\title{
Strongly Enhanced Antibacterial Action of Copper Oxide Nanoparticles with Boronic Acid Surface Functionality
}

\author{
Ahmed F. Halbus, , ${ }^{\text {ab }}$ Tommy S. Horozov, ${ }^{\text {a Vesselin N. Paunov }}{ }^{\mathrm{a} *}$ \\ ${ }^{\text {a }}$ Department of Chemistry and Biochemistry, University of Hull, Hull, UK; \\ ${ }^{\mathrm{b}}$ Department of Chemistry, College of Science, University of Babylon, Hilla, IRAQ.
}

KEYWORDS CuONPs; copper oxide, antibacterial nanoparticles, E. coli, R. rhodochrous, 4-Hydroxyphenylboronic acid; boronic acid, sugar, carbohydrates.

\begin{abstract}
Copper oxide nanoparticles (CuONPs) have been widely recognized as good antimicrobial agents but are heavily regulated due to environmental concerns of their post use. In this work, we have developed and tested a novel type of formulation for copper oxide (CuONPs) which have been functionalized with (3-Glycidyloxypropyl)trimethoxysilane (GLYMO) to allow further covalent coupling of 4-Hydroxyphenylboronic acid (4-HPBA). As the boronic acid (BA) groups on the surface of CuONPs/GLYMO/4-HPBA can form reversible covalent bonds with the diols groups of glycoproteins on the bacterial cell surface, they can strongly bind to the cells walls resulting in a very strong enhancement of their antibacterial action which is not based on electrostatic adhesion. SEM and TEM imaging revealed that 4-HPBA-functionalized nanoparticles could accumulate more on the cell surface than non-functionalized ones. We demonstrate that the CuONPs with boronic acid surface functionality are far superior antibacterial agents compared to bare CuONPs. Our results showed that, the antibacterial impact of the 4-HPBA functionalized CuONPs on Rhodococcus rhodochrous (R. rhodochrous) and Escherichia coli (E.coli) is one order of magnitude higher than that of bare CuONPs or CuONPs/GLYMO. We also observed a marked increase of the 4-HPBA functionalized CuONPs antibacterial action on these microorganisms at shorter incubation times compared with the bare CuONPs at the same conditions. Significantly, we show that the cytotoxicity of CuONPs functionalized with 4-HPBA as an outer layer can be controlled by the concentration of glucose in the media and that the effect is reversible as glucose competes with the sugar residues on the bacterial cell walls for the BA-groups on the CuONPs. Our experiments with human keratinocyte cell line exposure to CuONPs/GLYMO/4-HPBA indicated lack of measurable cytotoxicity at particle concentration which are effective as antibacterial agent for both $R$. rhodochrous and E.coli. We envisage that formulations of CuONPs/GLYMO/4-HPBA can be used to drastically reduce the overall $\mathrm{CuO}$ concentration in antimicrobial formulations while strongly increasing their efficiency.
\end{abstract}

\section{INTRODUCTION}

Spreading of antimicrobial resistance among common bacterial pathogens, bacterial infections, including antibiotic-resistant infections, have recently drawn much attention. ${ }^{1} \mathrm{~A}$ range of colloidal particles are being extensively studied in various antimicrobial applications due to their small size to volume ratio and ability to exhibit a wide spectrum of antibacterial action. ${ }^{2-5}$ Antibacterial NPs could bypass the increasing rates of antibiotic resistance by attacking and destroying the bacteria in other ways. ${ }^{6}$ Surface functionalization of nanoparticles is vital for controlling their properties and interactions with molecules and ligands of relevance for biomedical applications, in addition to their susceptibility to undergo a transformation in environmental and biological systems. ${ }^{45,46}$ Considerable efforts have been devoted to the development of surface modifiers that can offer not only stability but also better control of the interaction between nanoparticles and biological membranes in order to obtain more biocompatible materials. ${ }^{47}$ For example,
Al-Awady et al. ${ }^{44}$ produced polyelectrolyte-coated titania nanoparticles with up to 4 layers of polyelectrolytes of alternating charge (PSS and PAH) using the layer-by-layer technique. They showed that the antimicrobial properties of polyelectrolyte-coated nanoparticles alternate with the surface charge for the particles with cationic outer layer (or bare nanoparticles) being much more effective antimicrobials than the ones with an outer layer of anionic polyelectrolyte. The anionic nanoparticles (NPs/PSS and NPs/PSS/PAH/PSS) showed much lower activity towards than the cationic ones, NPs/PSS/PAH and the bare NPs, respectively. These authors suggested that the decrease of antimicrobial action can be explained by the poor adhesion of the anionic nanoparticles (NPs/PSS and NPs/PSS/PAH/PSS) to the cell walls due to their electrostatic repulsion. In contrary, the enhancement of the antimicrobial effect for cationic nanoparticles (bare NPs and $\mathrm{NPs} / \mathrm{PSS} / \mathrm{PAH}$ ) is due to the amplification of the particle-cell electrostatically driven adhesion. ${ }^{2,35}$ Perreault and co-workers have exhibited that polymer coated (polystyrene-co-butyl

This document is the Accepted Manuscript version of a Published Work that appeared in final form in ACS Applied Materials \& Interfaces, copyright (C) American Chemical Society after peer review and technical editing by the publisher. To access the final edited and published work see https://pubs.acs.org/doi/10.1021/acsami.8b21862 
acrylate) CuONPs showed increased cellular uptake and toxicity in the green alga $C$. reinhardtii. The ascorbate and citrate surface layers are well known for their anti-oxidant properties and are used as reducing agents as well as negatively charged stabilizers of nanoparticles synthesis and dispersion. ${ }^{48}$

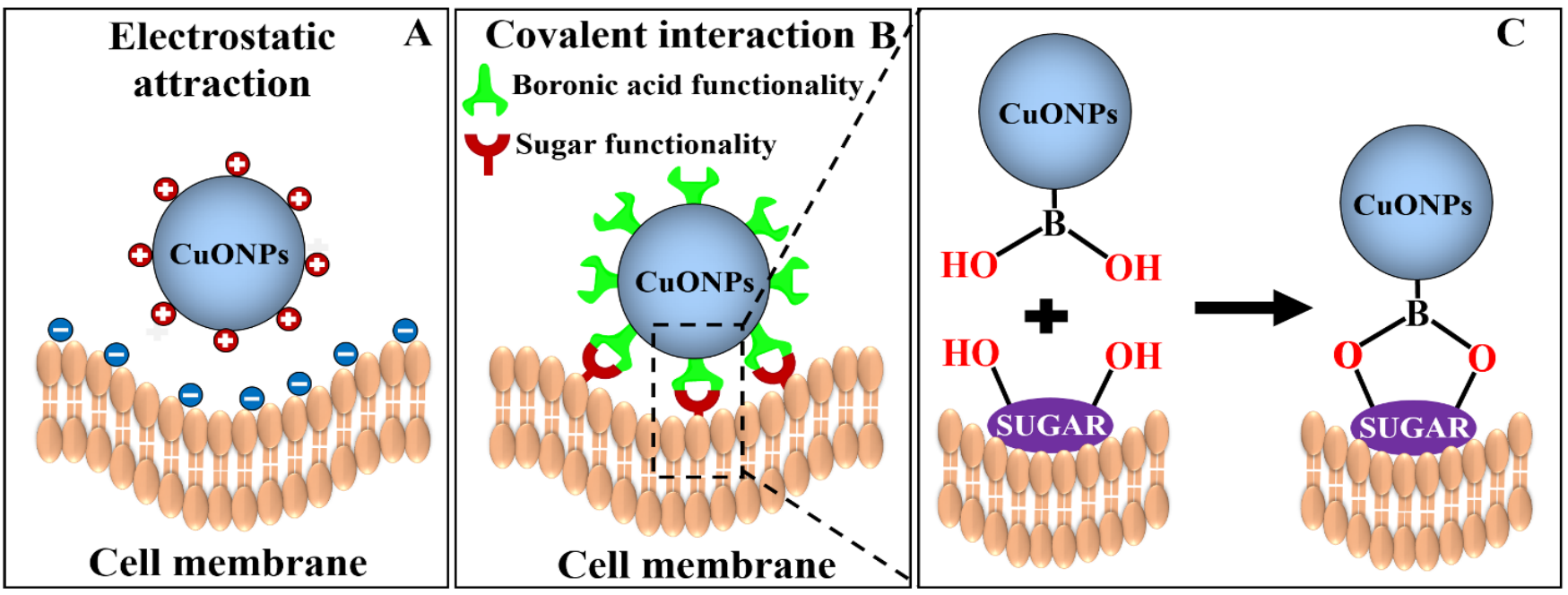

Figure 1. The perceived attachment mechanisms of CuONPs to the bacterial cell membranes. (A) Electrostatic attraction between bare CuONPs and the cells; (B) covalent bonding between CuONPs/GLYMO/4-HPBA and the cells. (C) The interaction between the CuONPs with boronic acid surface functionality and the sugar groups on the surface of the bacterial cell wall.

Líbalová et al. have evaluated the cytotoxicity of a panel of CuONPs with various surface modifications such as cationic polyethylenimine (PEI), neutral polyvinylpyrrolidone (PVP), sodium ascorbate (ASC) and anionic sodium citrate (CIT), versus the pristine bare CuONPs, using a murine macrophage cell line. The results from their work suggest that the PEI-coated CuONPs were found to be the most cytotoxic. Líbalová et al. have also reported that the ascorbate-coated CuONPs, which were found to be the least cytotoxic, produced lower levels of ROS in comparison to bare nanoparticles. ${ }^{46}$

CuONPs have been widely used as a dopant for semiconductors, chemical sensors, supported heterogeneous nano-catalysts, coating material and in anti-cancer treatments but their functional properties have been proven essential for their applications in biological research. ${ }^{7,8}$ Lazary and coworkers have stated that CuONPs have been widely used in hospitals as anti-microbial agents due to their ability to kill more than $99.9 \%$ of both Gram-negative and Gram-positive bacteria within 2 hours of treatment of various surfaces. It has been found that the use of $\mathrm{CuO}$ in this way has radically decreased the occurrence of hospital-acquired infections and the costs associated with health care. A non-intravenous approach to utilizing CuONPs in bed sheets is a very exciting innovation as the particles can decrease microbial attachment and therefore limit hospital acquired infections. ${ }^{9}$ By synthesizing a hybrid inorganic/organic species in the form of a $\mathrm{Cu}$-chitosan nanoparticle, Usman et al. ${ }^{10}$ have found that their antimicrobial action is highly effective when the coated particles have a size range of $2-350 \mathrm{~nm}$. The same research team has evaluated the antibacterial and antifungal activities of these nanoparticles on different microorganisms, including methicillin-resistant Staphylococcus aureus, Salmonella choleraesuis, Candida albicans, Pseudomonas aeruginosa, and Bacillus subtilis. The results from their work have shown that the highly effective Cu-chitosan particles are very active as antimicrobial agents in anaerobic conditions. Note that $\mathrm{Cu}$ nanoparticles have the ability to rapidly oxidize, which limits their applications as antimicrobials when used in aerobic conditions. $^{10,11}$ Katwal and others have developed a new CuONPs preparation route by using electrochemical methods and demonstrated that they can control the CuONPs morphologies. ${ }^{12} \mathrm{CuO}$ particles can be produced in various shapes and sizes, and can provide enhanced antibacterial activity against several pathogenic strains. Mahapatra et al. ${ }^{11}$ tested the antibacterial action of CuONPs towards Klebsiella pneumoniae, Salmonella paratyphi, Shigella strains and Pseudomonas aeruginosa and showed that the nanoparticles have been efficient against these bacteria. They envisaged that CuONPs can cross through the bacterial cell membrane and affect vital enzymes in the bacteria cytoplasm leading to their death. It has also been shown that CuONPs are not cytotoxic on some human cells (e.g. HeLa cell line). Azam et al. ${ }^{13}$ have also reported that the activity of $\mathrm{CuO}$ based nanoparticles is dependent on their particle size when used as an antibacterial agent. In their study, they examined two Gram-negative bacteria (E. coli and $P$. aeruginosa) and two Gram-positive bacteria (B. subtilis and $S$. aureus). It was found that CuONPs exhibited inhibitory effects towards both groups of bacteria, which clearly depended on their stability, particle size and concentration when incubated with the bacterial culture. They concluded that the CuONPs can limit the bacterial growth by interacting with nanometric pores that exist on the cell membranes of most microorganisms. Ahamed and co-workers discovered that CuONPs with a size of $\sim 23 \mathrm{~nm}$ had significant 
antimicrobial action towards various bacterial strains (Klebsiella pneumoniae, Pseudomonas aeruginosa, Escherichia coli, Enterococcus faecalis, Shigella flexneri, Salmonella typhimurium, Staphylococcus aureus, and Proteus vulgaris). Escherichia coli and Enterococcus faecalis showed the highest sensitivity to CuONPs while Klebsiella pneumoniae
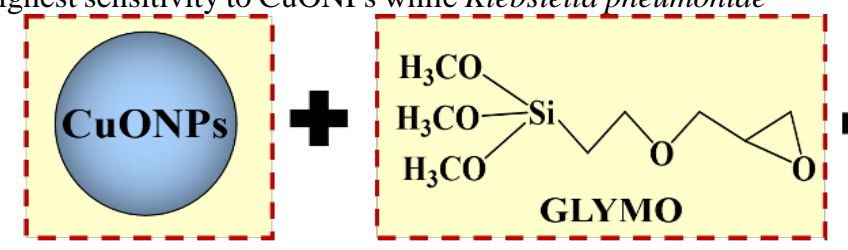

\section{Boronic acid functionality}

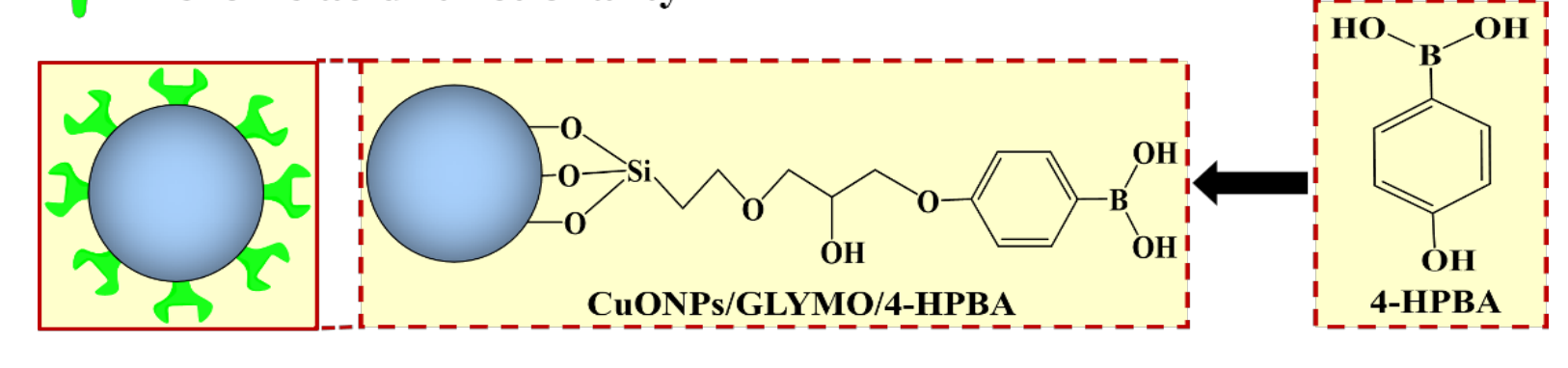

was resistant to this treatment. ${ }^{2,7,8}$ Note that the bare CuONPs are cationic at neutral $\mathrm{pH}$ and can adhere to the negatively charged bacterial cell walls only by electrostatic interactions.

Figure 2. The schematic of the surface functionalization of CuONPs with GLYMO and 4-HPBA.

The average size of CuONPs is also essential for their potential antimicrobial activity, as smaller nanoparticles have higher portability and ability to potentially penetrate and relocate between the bacterial cell compartments. This makes them very effective antimicrobial agents. However, electrostatic adhesion can be easily disabled by the presence of another type of anionic substances in the solution, e.g. organic acids, albumins, surfactants, polymers and others. It impacts the nanoparticle interactions with different biomolecules, for example, carbohydrates and proteins which can be adsorbed on the particles and form a corona of different surface properties to that of the original nanoparticles. This is the likely reason why CuONPs can quickly lose their antimicrobial activity in biological fluids as well as in formulations that contain anionic polyelectrolytes and surfactants.

Here we engineered CuONPs with boronic acid surface functionality in an attempt to design a non-electrostatic mechanism for their attachment to the bacteria which was expected to amplify their accumulation on the cell walls despite the presence of other anionic species. This is shown schematically in Figure 1. Our idea here is to introduce boronic acid (BA) surface groups on the CuONPs which are able to covalently bind to various glycoproteins and carbohydrates that are abundant on the bacterial cell walls.

Boronic acid has been used before in chemosensor applications due to its high sensitivity for sugar determination. ${ }^{14}$ An attractive feature of the BA surface functionality that makes it very effective for biomedical applications is their perceived absence of toxicity ${ }^{15}$ despite its ability to form reversible covalent complexes with diols. ${ }^{16,17}$ The binding of BA to sugars is very sensitive to the sugar concentration, however, it is undiscriminating and will therefore bind to any diol containing compounds. ${ }^{18} \mathrm{BA}$ has also been discussed as a promising tools for the quantification of the total content of bacteria. ${ }^{19-21} \mathrm{BA}$ surface groups can covalently bind to saccharides and form boronic esters. ${ }^{22-24}$

We used $R$. rhodochrous and E.coli as model bacteria species to examine the antibacterial activity of the 4-HPBA functionalized CuONPs. The current work was carried out with CuONPs, CuONPs/GLYMO and CuONPs/GLYMO/4-HPBA to investigate the impact of (i) the nanoparticle concentration, and (ii) the zeta potential and particle size on the viability of $R$. rhodochrous and E.coli at different exposure times. The novelty of our work is that the antibacterial activity of CuONPs functionalized with 4-HPBA is not based on electrostatic adhesion to the bacterial cells and therefore could potentially be used in complex biological environment. Significantly, the functionalization of the CuONPs with 4-HPBA groups as an outer monolayer should lead to their covalent attachment on the sugar $(\mathrm{OH})$ groups on the membrane surface, thus bringing the CuONPs in very close proximity to the bacterial cell membrane and increasing their efficiency (Figure $1 \mathrm{~B}$ and $1 \mathrm{C}$ ). We also examined the toxicity of both bare CuONPs and functionalized CuONPs on human keratinocytes.

\section{MATERIALS AND METHODS}

\section{Materials}

We used copper (II) chloride (99\%, Sigma Aldrich) as a precursor in the synthesis of CuONPs by the direct precipitation method. Sodium hydroxide (99.6\%, Fisher, UK) was used as a precipitating agent to synthesise CuONPs. (3glycidyloxypropyl) trimethoxysilane (GLYMO) and 4hydroxyphenylboronic acid (4-HPBA) were purchased from Sigma Aldrich. BacTiter-Glo (BTG) microbial cell viability assay was delivered by Promega, UK. Escherichia coli, sourced 
from Thermofisher (Invitrogen MAX Efficiency ${ }^{\mathrm{TM}}$ DH10B ${ }^{\mathrm{TM}}$ ) was kindly provided for our antibacterial tests by Prof J. Rotchell's group at the University of Hull, UK. R. rhodochrous was supplied by Blades Biological Ltd., UK. Deionized water purified by reverse osmosis and ion exchange with a Milli-Q water system (Millipore, UK) was used in all our studies. Its surface tension was $71.9 \mathrm{mNm}^{-1}$ at $25^{\circ} \mathrm{C}$, with measured resistivity more than $18 \mathrm{M} \Omega \mathrm{cm}^{-1}$.
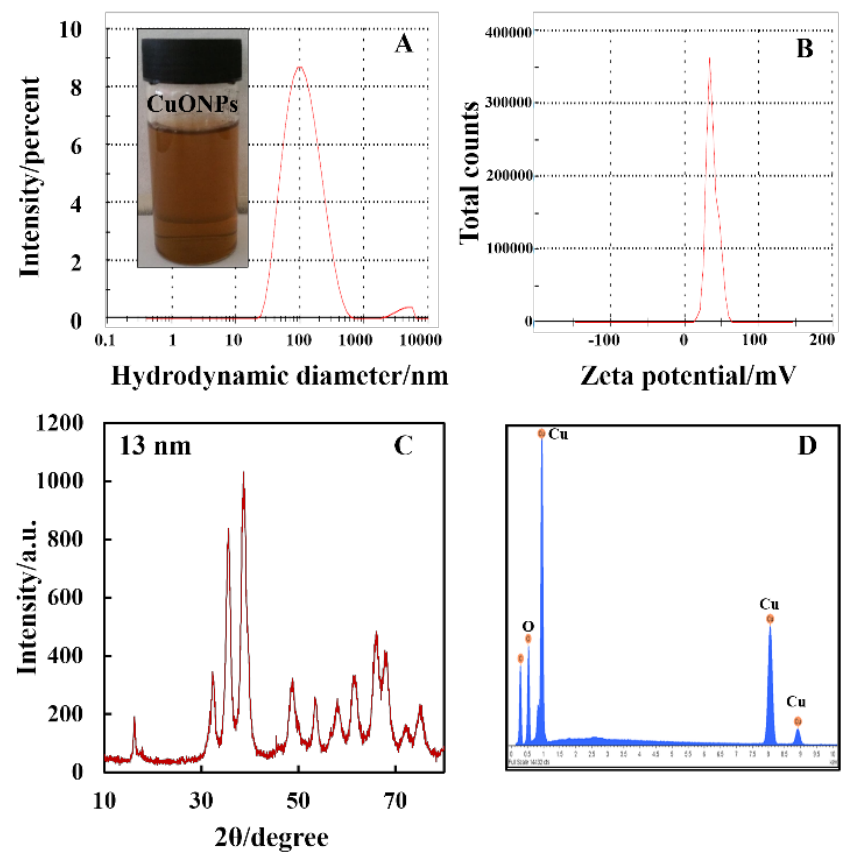

Figure 3. Plots of (A) particle size and (B) zeta potential distribution of $\mathrm{CuONPs}$ produced by annealing at $100^{\circ} \mathrm{C}$. The size and zeta potential of CuONPs was measured utilizing the Malvern Zetasizer Nano ZL at room temperature with the average data of three runs. (C) XRD pattern of CuONPs annealed at $100{ }^{\circ} \mathrm{C}$. The largest peak in the XRD results was used to determine the crystallite size. (D) EDX spectrum of CuONPs.

\section{Methods}

Synthesis of CuONPs. In first stage of the preparation, $3.0 \mathrm{~g}$ of copper (II) chloride $\left(\mathrm{CuCl}_{2}\right)$ was dissolved in $160 \mathrm{~mL}$ of ethanol. $1.8 \mathrm{~g}$ of sodium hydroxide $(\mathrm{NaOH})$ was dissolved in 50 $\mathrm{mL}$ ethanol. The $\mathrm{NaOH}$ solution was added dropwise to $\mathrm{CuCl}_{2}$ solution under constant stirring at room temperature. During the course of the reaction, the color of the solution turned from green to greenish blue and lastly to black. This black precipitate was copper hydroxide, $\mathrm{Cu}(\mathrm{OH})_{2}$ (see Figure S1) which was centrifuged, washed with ethanol and deionized water, and dried at $60{ }^{\circ} \mathrm{C}$ in the electric furnace. In order to produce CuONPs, the sample of dry $\mathrm{Cu}(\mathrm{OH})_{2}$ was annealed at different temperatures, $100{ }^{\circ} \mathrm{C}, 200{ }^{\circ} \mathrm{C}, 300{ }^{\circ} \mathrm{C}, 400{ }^{\circ} \mathrm{C}, 500{ }^{\circ} \mathrm{C}$ and 600 ${ }^{\circ} \mathrm{C}$ (see Figures S2 and S3) followed by grinding to obtain $\mathrm{CuO}$ in powdered form. ${ }^{25} \mathrm{CuONPs}$ were produced by dispersing $\mathrm{CuO}$ in Milli-Q water at pH 6 via a sonication (Branson 450, $5 \mathrm{~mm}$ tip, $400 \mathrm{~W}$ maximum power) at $40 \%$ of the maximum power for 10 minutes (2 s ON - 2 s OFF pulse time).
Surface Functionalization of CuONPs by GLYMO and 4HPBA. A sample of $0.1 \mathrm{~g}$ of CuONPs was dispersed into deionized water (100 mL, pH 6-6.5). The suspension was stirred for 1 hour and 0.1 wt\% of GLYMO were added. The reaction mixture was stirred for a further 24 hours, then the unreacted GLYMO was removed by centrifugation and washing with deionized water three times. The process is analogous to the APTES functionalization of other inorganic nanoparticles ${ }^{26}$ but in our case GLYMO brings epoxy-ring as a terminal group. This functionality has not been reported before for CuONPs. The GLYMO- functionalized CuONPs pellet was then re-dispersed in $100 \mathrm{~mL}$ of deionized water and mixed dropwise with $0.1 \mathrm{~g}$ of 4 -HPBA dissolved in $100 \mathrm{~mL}$ of ethanol solution. The mixture was shaken for 2 hours, then washed and centrifuged three times with ethanol at $10000 \mathrm{rpm}$ for 30 minutes. The CuONPs/GLYMO/4-HPBA produced were finally re-dispersed in $100 \mathrm{~mL}$ of deionized water. ${ }^{27-29}$ The chemistry of the process of surface functionalization of CuONPs with phenyl boronic acid is shown in Figure 2.

Characterization of the Surface Functionalized CuONPs. The particle size and the zeta potential of the surface functionalized CuONPs (with GLYMO and 4-HPBA) was examined by dynamic light scattering (DLS) using the Malvern Zetasizer Nano ZL instrument. The zeta potential values of the surface functionalized CuONPs were determined after dispersing the CuONPs samples in deionized water using an ultrasonic probe. After that, a range of CuONPs suspensions with $\mathrm{pH}$ from 3 to 12 was made by using $0.1 \mathrm{M} \mathrm{HCl}$ and $0.1 \mathrm{M}$ $\mathrm{NaOH}$ and adding two drops of $0.01 \mathrm{M} \mathrm{NaCl}$ into each sample $(10 \mathrm{~mL})$. All measurements have been done at room temperature and the results reported are an average of 3 runs.

Antibacterial Activity of Bare and Surface Functionalized CuONPs on $E$. coli and $R$. rhodochrous. 10 $\mathrm{mL}$ of the bacteria culture was centrifuged and washed three times with deionized water for 4 minutes at $4000 \mathrm{rpm}$, and redispersed in $100 \mathrm{~mL}$ deionized water. Then, $5 \mathrm{~mL}$ of the washed bacteria were incubated with a series of $5 \mathrm{~mL}$ aliquots of the CuONPs suspension at various particles concentrations. The number of bacteria was measured directly after removing the excess nanoparticles from the bacteria dispersion. Then, $1 \mathrm{~mL}$ of each bacteria suspension was washed and re-suspended in 1 $\mathrm{mL}$ deionized water. $100 \mu \mathrm{L}$ aliquot of the washed bacteria suspension was then incubated with $100 \mu \mathrm{L}$ of BTG reagent in a white opaque 96-well microplate with solid flat bottom, and after that shaken for 30 seconds, and incubated at $30{ }^{\circ} \mathrm{C}$ for 5 minutes. The relative luminance was measured as a function of the incubation time and used to calculate the fraction of viable bacteria upon exposure to various concentrations of CuONPs. We did the same experiments with CuONPs functionalized with GLYMO as well as ones functionalized with GLYMO and 4HPBA at various particle concentrations.

Colony Forming Units assessment for antimicrobial assay. The bacteria were grown overnight in sterilized LB medium at $37{ }^{\circ} \mathrm{C}$ to produce viable colonies. Bacterial cells were pelleted down by centrifugation at $5000 \mathrm{rpm}$ for 10 minutes followed by washing (twice) with $0.85 \mathrm{w} / \mathrm{v} \%$ serial saline until an optical density of $0.08-0.12$ at $625 \mathrm{~nm}$ was obtained using a spectrophotometer. These adjusted bacterial saline suspensions were then diluted 1:150 into LB to yield 
starting concentrations between $5 \times 10^{5}-1 \times 10^{6}$ colony forming units per $\mathrm{mL}(\mathrm{CFU} / \mathrm{mL})$. Then flasks $250 \mathrm{~mL}$ containing LB medium $100 \mathrm{~mL}$ with different concentrations of the bare CuONPs and surface functionalized of CuONPs with GLYMO and 4-HPBA were inoculated with an equal volume of the bacterial suspension. Flasks containing bacterial cells and media without nanoparticles were used as control. All the flasks were incubated for $10 \mathrm{~min}, 1$ hour and 6 hours in a shaker at 37 ${ }^{\circ} \mathrm{C}$ with $140 \mathrm{rpm}$. After that, the serial dilutions were made of all the treated samples including control and $100 \mu \mathrm{L}$ of each were homogeneously spread on LB agar plates for colony forming unit (CFU). The growth rate of bacterial cells interacting with the nanoparticles was determined from a plot of the CFU/mL versus concentrations. The time-kill assay was repeated in three independent experiments

Zeta Potential Measurements of E.coli after Treatment with CuONPs. The changes in the surface charge of the bacteria after incubation with the bare and the surface modified CuONPs were determined by a Zetasizer nano ZL instrument (Malvern, UK) at nanoparticle concentrations of $0,5,10,15$, 20 , and $25 \mu \mathrm{g} \mathrm{mL}{ }^{-1}$. Equal aliquots of the cell culture was used to measure the average zeta potential value of the bacteria after being incubated with the particles. All measurements were done in triplicates.

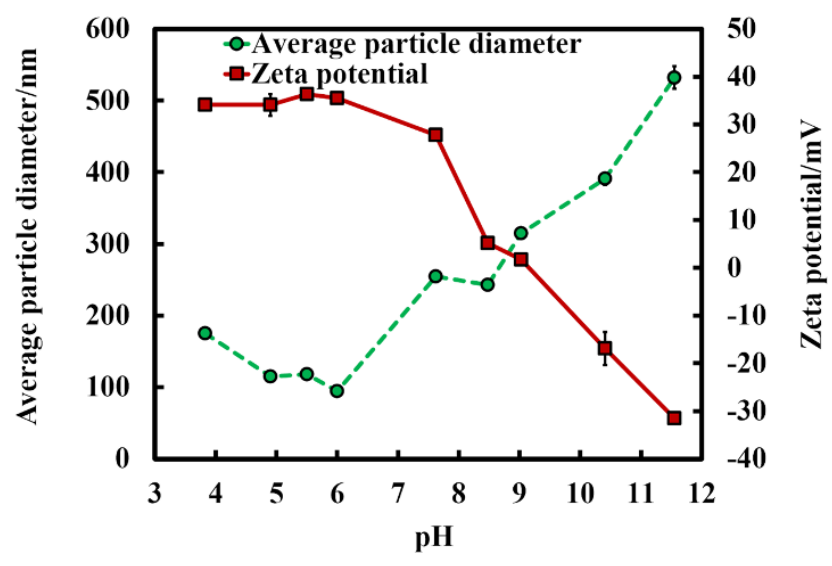

Figure 4. Zeta potential and particle diameter of bare CuONPs versus pH of the aqueous suspension.

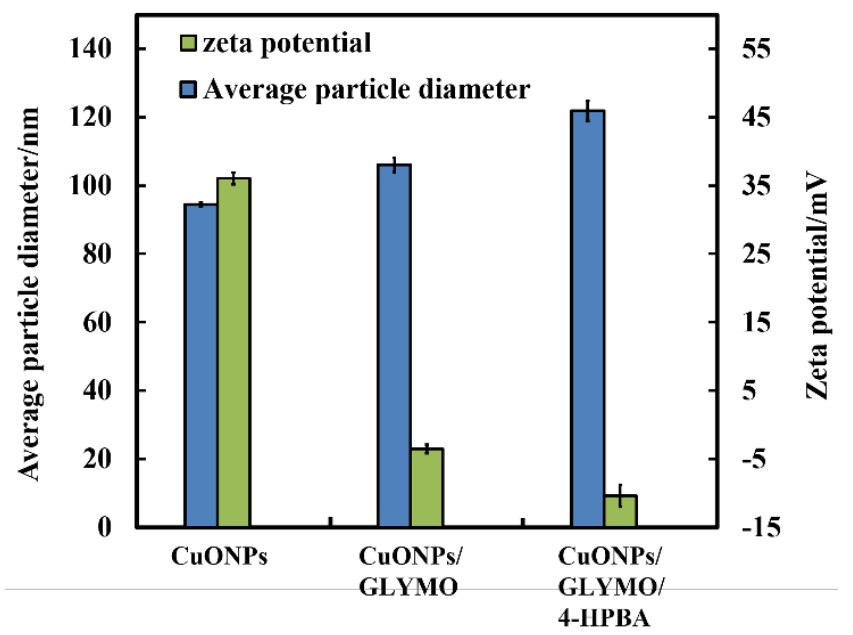

Figure 5. Zeta potential and hydrodynamic diameter of the bare and surface modified CuONPs with GLYMO and 4HPBA, measured at room temperature at pH 6 (error bars are standard deviations).

SEM and TEM Sample Preparation Protocol for E.coli and $R$. rhodochrous after Exposure to HPBAFunctionalized CuONPs. After incubation with the HPBAsurface modified CuONPs, the E.coli and $R$. rhodochrous were fixed with $2.5 \%$ glutaraldehyde at room temperature for two hours in $0.1 \mathrm{M}$ cacodylate buffer $\mathrm{pH}$ 7.2. These samples were then post-fixed in $1 \%$ osmium tetroxide for one hour, and dehydrated in a range of ethanol-water mixtures with increasing ethanol content from 50 vol\% up to 100 vol\% followed by critical point drying. After incubation with CuONPs, the bacterial cells were prepared for TEM imaging using the following procedure. The bacteria were washed with deionized water to remove the excess of CuONPs at $500 \mathrm{rpm}$ and then fixed in $2 \mathrm{wt} \%$ glutaraldehyde for one hour at room temperature followed by treatment with $1 \mathrm{wt} \%$ osmium tetroxide for one hour. Then, the samples were incubated for one hour with 2.5 $\%$ uranyl acetate and washed with aqueous ethanol solutions of increasing concentration, as described above. 


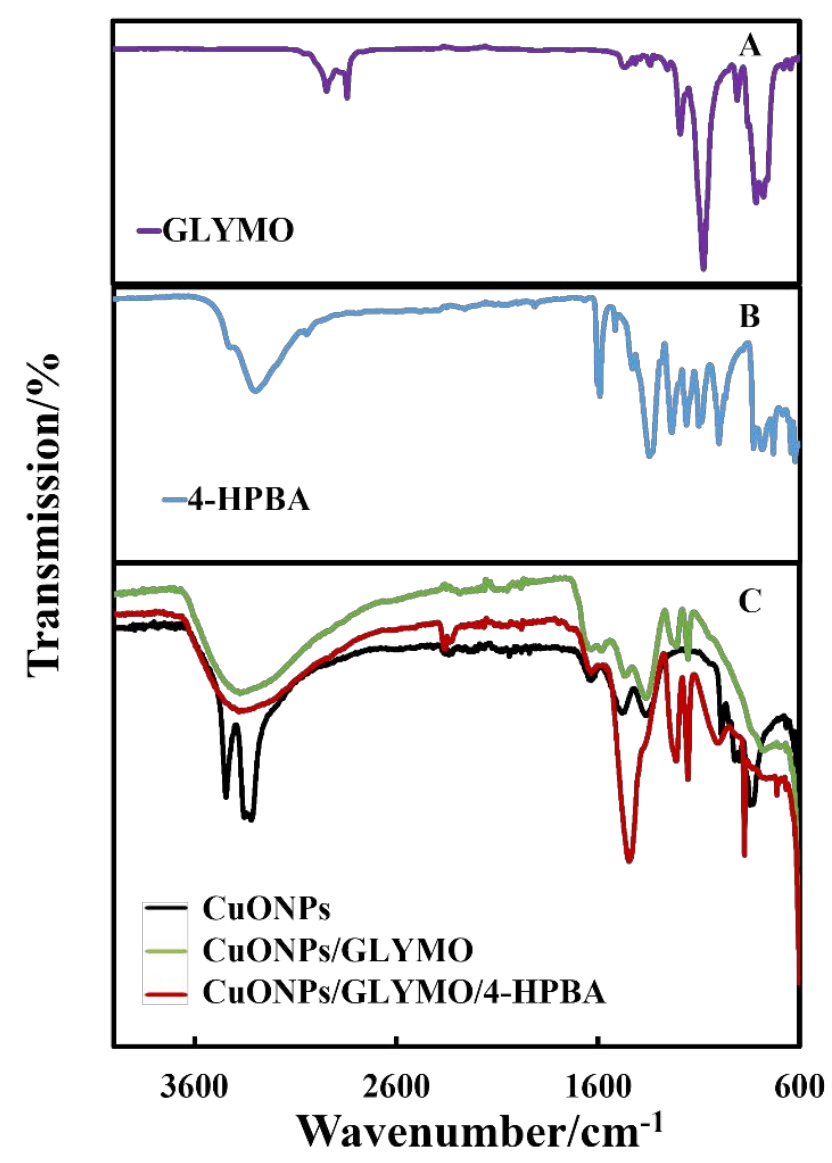

Figure 6. The FTIR spectra of (A) pure GLYMO, (B) pure 4-HPBA and $(C)$ the bare and functionalized CuONPs.

After standard dehydration, the bacterial samples were embedded in fresh epoxy/Araldite at $60{ }^{\circ} \mathrm{C}$ for 2 days, left for 2 days at room temperature and sectioned with an ultramicrotome. Bacteria samples before and after the nanoparticle treatment were imaged by SEM and TEM.

\section{Cytotoxicity Assay of Bare and Surface Functionalized CuONPs on HaCaT Cells.}

HaCaT cell line culture (immortalized human keratinocytes) was kindly provided by the Skin Research Group at St James
University Hospital at Leeds. The cells were cultured in highglucose DMEM media supplemented with 10\% Fetal Bovine Serum (FBS, Labtech, UK) and 1\% antibiotics (Penicillin Streptomycin, Lonza, UK) and placed in an incubator $\left(37^{\circ} \mathrm{C}\right.$, $5 \% \mathrm{CO}_{2}$ ). After reaching $70 \%$ confluence, HaCaT cells were carefully washed with PBS for 10 seconds then incubated with $0.25 \%$ Trypsin-EDTA (1X, Lonza, UK) to detach the cells from their support after 5 minutes. Its action was neutralized by adding complete DMEM medium before a centrifugation at $400 \times \mathrm{g}$ for 4 minutes. An $25 \mathrm{~mL}$ aliquot of the HaCaT cells culture ( $\sim 75000$ cells $\left.\mathrm{mL}^{-1}\right)$ were washed three times from the culture media via centrifuged, and re-dispersed with $25 \mathrm{~mL}$ PBS. Then, $2.5 \mathrm{~mL}$ aliquots of this HaCaT cells suspension were incubated with a series of $2.5 \mathrm{~mL}$ aliquots of aqueous dispersions of bare and surface functionalized CuONPs at different concentrations. Likewise, a control sample of the $\mathrm{HaCaT}$ cells was treated at the similar conditions without exposure to any nanoparticles. After that, $1 \mathrm{~mL}$ of the solution HaCaT was taken from each addressed sample with nanoparticles, washed with PBS to remove the excess of nanoparticles via centrifuged at $400 \times \mathrm{g}$ for 4 minutes. The $\mathrm{HaCaT}$ was re-suspended in $1 \mathrm{~mL}$ of PBS, then two drops of FDA solution in acetone was added to each sample and mixed together for 15 minutes followed by triple washing with PBS by centrifugation at $400 \times \mathrm{g}$ for 4 minutes. Finally, a microplate reader was utilized to assay the HaCaT cell viability.

\section{RESULTS AND DISCUSSION}

Characterization of CuONPs. We studied the mean particle hydrodynamic diameter and zeta potential of bare and functionalized CuONPs. The results for the non-functionalized particles are presented in Figures S4 and S5 (ESI). The bare CuONPs average particle hydrodynamic diameter was about 93 $\mathrm{nm}$ while their average zeta potential was around $+37 \mathrm{mV}$, i.e. the non-functionalized (bare) $\mathrm{CuO}$ nanoparticles are cationic at $\mathrm{pH} 6$ (see Figures $3 \mathrm{~A}$ and $3 \mathrm{~B}$ ). Since the CuONPs are photoactive, there was a concern that the GLYMO/HPBA functionality can potentially be affected by oxidation under the action of UV light. In order to check the stability of this coating against oxidation, we measured periodically the zeta potential of the CuONPs/GLYMO/4-HPBA over the course of 3 days while the samples were exposed to UV light. 

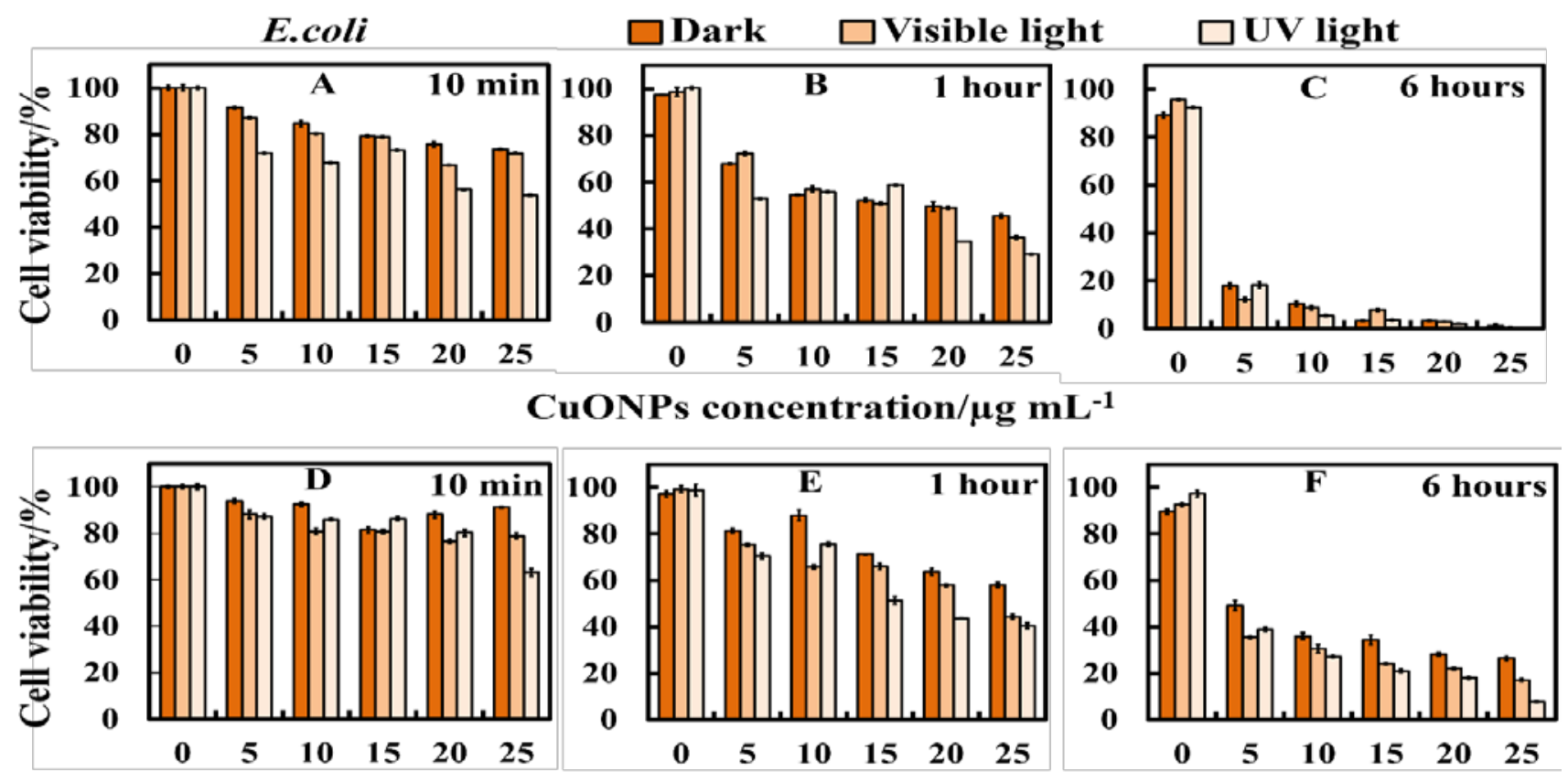

\section{CuONPs/GLYMO concentration/ $/ \mathrm{g} \mathrm{mL}^{-1}$}
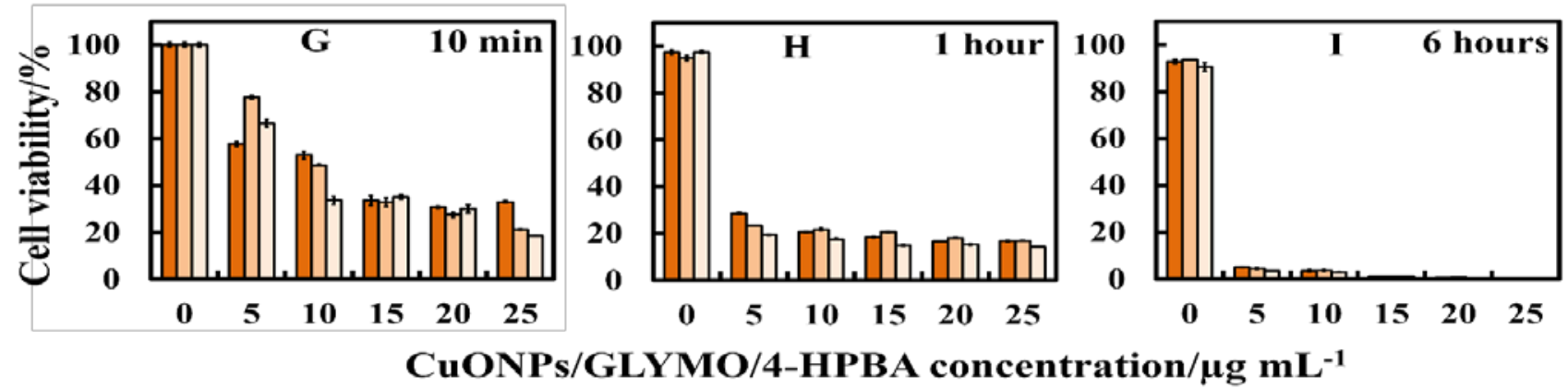

Figure 7. Comparison of the E.coli viability at various concentrations of the bare CuONPs (A - C), and surface functionalized of CuONPs with GLYMO (D - F) and 4-HPBA (G - I) in dark, visible and UV light conditions at different incubation times (shown). 

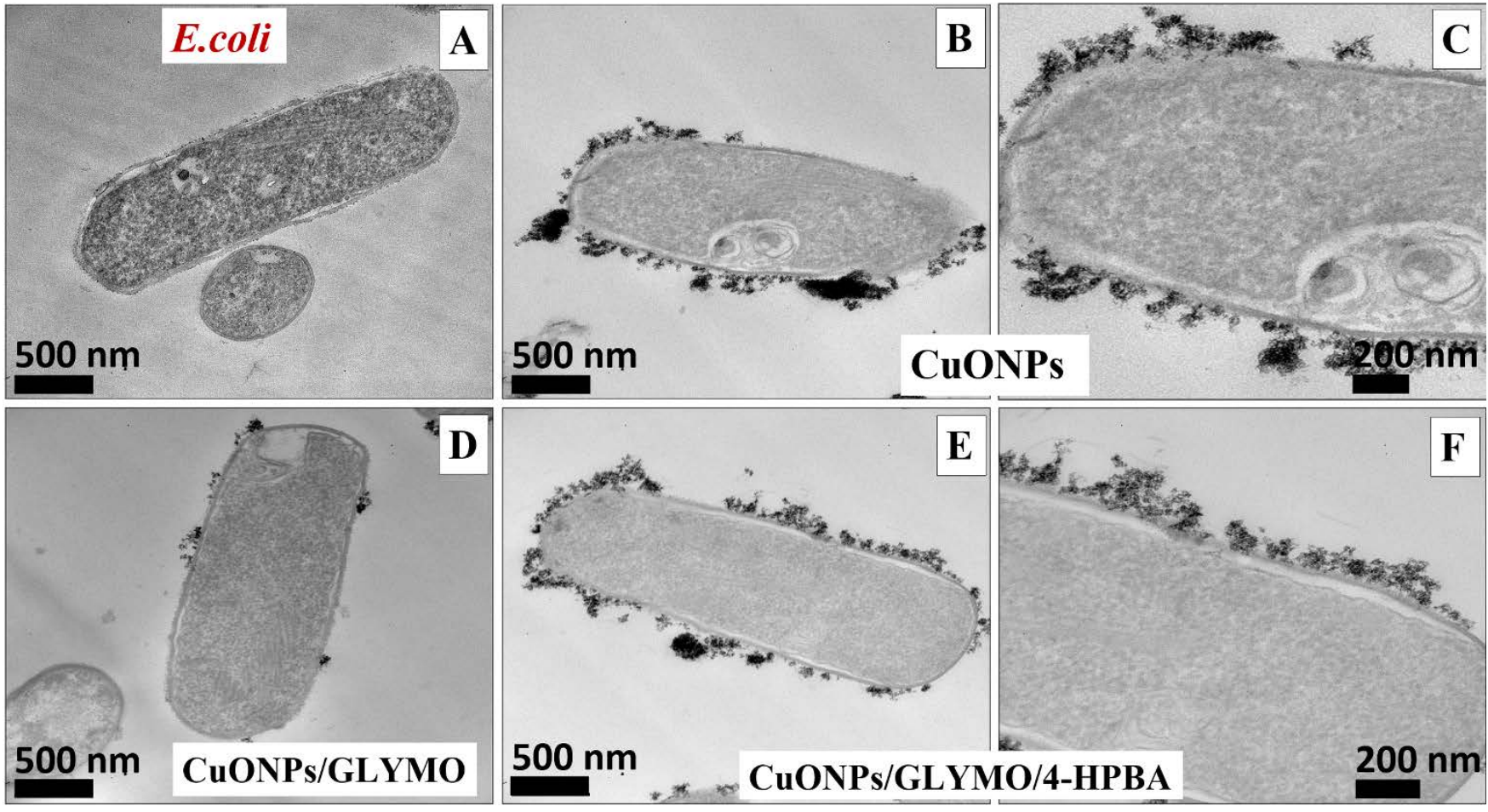

Figure 8. TEM images of E.coli at different magnifications: (A) before treatment, and (B, C) after treatment with $25 \mu \mathrm{g} \mathrm{mL}^{-1}$ bare CuONPs, (D) $25 \mu \mathrm{g} \mathrm{mL}{ }^{-1} \mathrm{CuONPs} / \mathrm{GLYMO}$ and (E, F) $25 \mu \mathrm{g} \mathrm{mL} \mathrm{mL}^{-1} \mathrm{CuONPs} / \mathrm{GLYMO} / 4-\mathrm{HPBA}$, all for 6 hours.

The results, presented in Figure S7 (ESI) indicate that the zetapotential of the functionalized CuONPs does not change, i.e. the coating is not prone to oxidation at these conditions and hence the particles preserve their functionality and antibacterial action. Figure 3C shows XRD pattern of CuONPs produced by the direct precipitation method and calcinated at $100{ }^{\circ} \mathrm{C}$. The diffraction peaks agree very well with the hexagonal structure of $\mathrm{CuO}$ according to the Joint Committee on Powder Diffraction Standards (JCPDS no.01-077-7716). No apparent impurities were detected, suggesting that CuONPs of high purity were prepared. The average crystal size of CuONPs calculated from XRD data using the Scherrer equation was about $13 \mathrm{~nm}$, i.e. much smaller than the hydrodynamic diameters of the CuONPs dispersed in deionized water. This indicates that the CuONPs in aqueous dispersions are aggregates of smaller crystallites. Figure 3D shows the EDX spectrum of CuONPs annealed at $100{ }^{\circ} \mathrm{C}$. The results confirm the presence of only copper $(\mathrm{Cu})$ and oxygen $(\mathrm{O})$ in the CuONPs samples and the data indicate that the nanoparticles were nearly stoichiometric with $73.3 \mathrm{wt} \% \mathrm{Cu}(0.804 \mathrm{keV})$ and $13.7 \mathrm{wt} \% \mathrm{O}(0.525 \mathrm{keV})$, respectively. There was no indication of any other elemental impurities in the EDX spectra, i.e. the copper-to-oxygen atomic ratio was $1: 1$ in the CuONPs, which is in agreement with the literature. ${ }^{30,31}$

Characterization of the CuONPs Surface Functionalized of with GLYMO and 4-HPBA. The zeta potential of bare CuONPs as a function of $\mathrm{pH}$ is shown in Figure 4. The isoelectric point (IEP) of the CuONPs (corresponding to the $\mathrm{pH}$ where the CuONPs have zero zeta potential) is at $\mathrm{pH}$ 9. We found that at $\mathrm{pH}$ values above the IEP, the CuONPs partially lost their colloid stability and formed larger aggregates (500 nm or bigger). To avoid the ambiguity related to the particle surface charge being influenced by $\mathrm{pH}$, we carried the antibacterial tests at $\mathrm{pH}$ between 5 and 6 (away from the IEP) to ensure that the particle size is around $100 \mathrm{~nm}$. The zeta potentials and hydrodynamic diameters of bare and functionalized CuONPs determined at $\mathrm{pH} 6$ are compared in Figure 5. One can see that the bare CuONPs dispersed in deionized water have the smallest hydrodynamic diameter $(94 \pm 3 \mathrm{~nm})$, while the diameter of surface-modified CuONPs varied between $106 \pm 6 \mathrm{~nm}$ (for CuONPs/GLYMO) and 121 $\pm 4 \mathrm{~nm}$ (for CuONPs/GLYMO/4HPBA). The zeta potential of bare CuONPs was positive while the two types of surface-modified CuONPs had small but negative zeta potential, ranging from around $-3 \pm 2 \mathrm{mV}$ (CuONPs/GLYMO) to $-10 \pm 2 \mathrm{mV}$ (CuONPs/GLYMO/4HPBA) (see Figure 5). The efficiency of the alkoxysilanemediated functionalization with GLYMO (and latter with 4HPBA) on CuONPs was examined by FTIR. The OH groups on the surface of the $\mathrm{CuO}$ nanoparticles are the reactive sites for the reaction with alkoxy silane groups of GLYMO. Figures 6A, 6B and 6C show normalized FTIR spectra of the bare CuONPs and those, surface modified with GLYMO or GLYMO/4HPBA. In the spectra of all CuONPs, the broad band between 400 and $800 \mathrm{~cm}^{-1}$ corresponds to $\mathrm{Cu}-\mathrm{O}-\mathrm{Cu}$. GLYMO contains two functional groups: epoxy and methoxysilyl, which can both hydrolyze and condensate. One can see that the epoxy band in FTIR spectra (Figure 6A) is preserved, while the intensity of Si$\mathrm{O}-\mathrm{Me}$ band is decreased. Moreover, the two bands of $\mathrm{OH}$ groups appear at $\sim 3300$ and $\sim 1640 \mathrm{~cm}^{-1}$ because of the hydrolysis of Si-O-Me groups. 

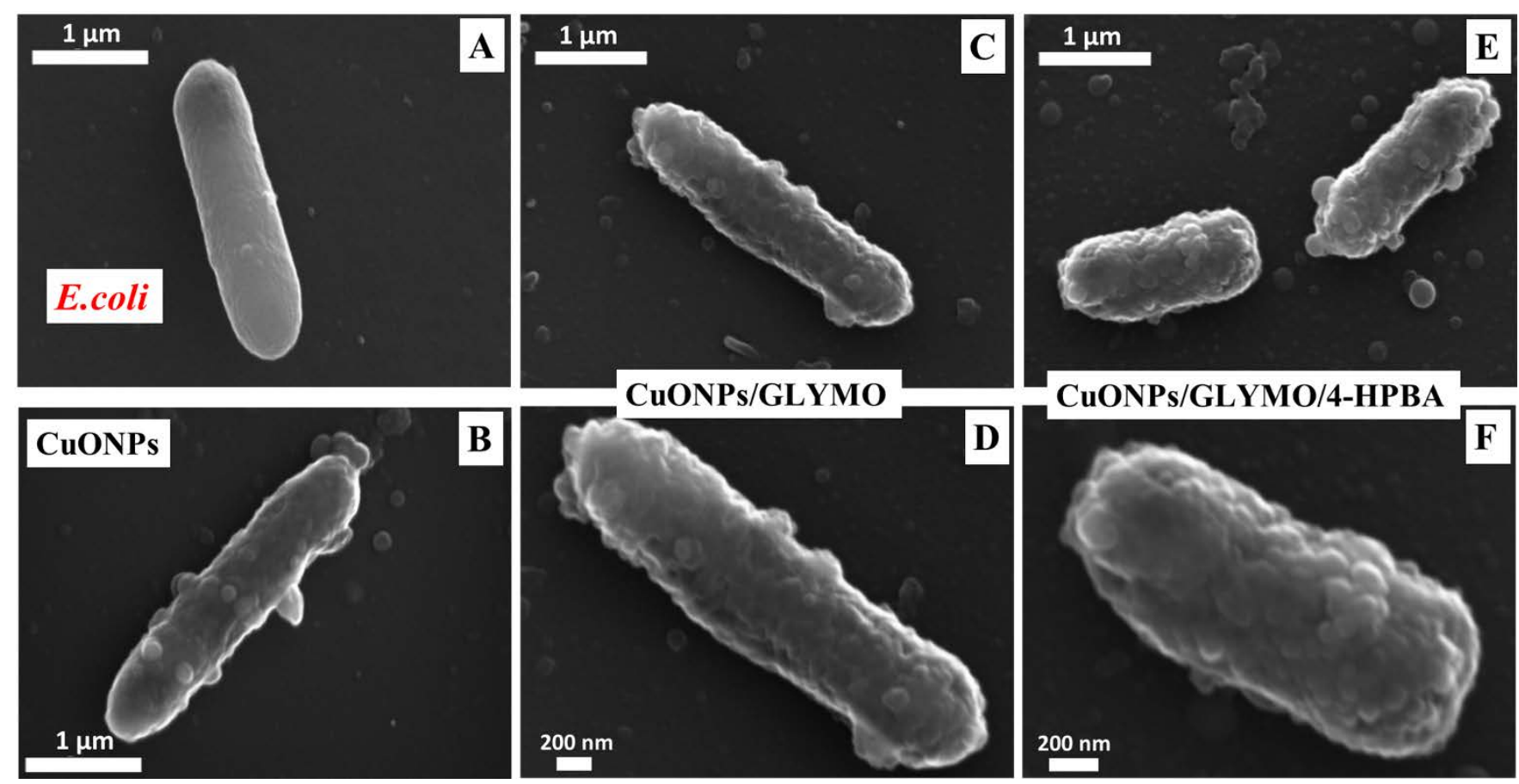

Figure 9. SEM images of E.coli after being incubated for 6 hours with bare CuONPs and CuONPs functionalized with GLYMO or

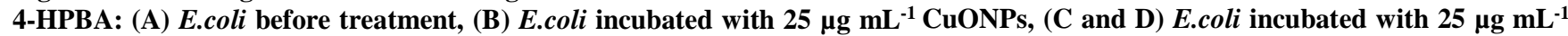
CuONPs/ GLYMO at different magnifications. (E and F) E.coli incubated with $25 \mu \mathrm{mL}^{-1} \mathrm{CuONPs} / \mathrm{GLYMO} / 4-\mathrm{HPBA}$ at different magnifications. Note the extensive build-up of (B) CuONPs and (E,F) CuONPs/GLYMO/4-HPBA on the E. coli cell walls.

Also a peak at $1050 \mathrm{~cm}^{-1}$ appears, which can be assigned to the formation of Si-O-Si groups. The comparison of the FTIR spectra of the bare and functionalized CuONPs samples show some new characteristic absorption peaks.

Figure 6C (CuONPs/GLYMO) shows a peak at $\sim 1200 \mathrm{~cm}^{-1}$ which refers to $\mathrm{Si}-\mathrm{O}-\mathrm{Me}$ groups. ${ }^{32}$ In the FTIR spectrum of the CuONPs/GLYMO/4-HPBA, the peak at about $3300 \mathrm{~cm}^{-1}$ could be attributed to the stretching vibration of $\mathrm{O}-\mathrm{H}$ groups. The peaks at $\sim 2500 \mathrm{~cm}^{-1}$ were assigned to the stretching and bending vibrations of $\mathrm{C}-\mathrm{H}$ groups. The bending of the aromatic $\mathrm{C}=\mathrm{C}$ groups could be also observed at $1490-1650 \mathrm{~cm}^{-1}$. The sharp peaks at around $1343 \mathrm{~cm}^{-1}$ and $1090 \mathrm{~cm}^{-1}$ could be assigned to the stretching vibrations $\mathrm{B}-\mathrm{O}$ and $\mathrm{C}-\mathrm{B}$ groups (Figure 6C). ${ }^{33,34}$

Antibacterial Activity of Surface Functionalized CuONPs against E.coli. We examined the antibacterial activity of CuONPs surface functionalized with GLYMO and 4-HPBA on E.coli at $\mathrm{pH}$ 6. Although the bare CuONPs are cationic below $\mathrm{pH} 9$, their functionalization with GLYMO resulted in weakly negatively charged CuONPs/GLYMO. Further functionalization with 4-HPBA also yielded negatively charged CuONPs/GLYMO/4-HPBA. The E.coli cells were extracted from the growth media and redispersed in deionized water and aliquots of this $E$. coli cultures were incubated with fixed concentration of the nanoparticles (i) under UV light, (ii) under visible light and (iii) in dark conditions.

The E.coli culture was incubated with CuONPs at different particle concentrations $\left(0,5,10,15,20\right.$ and $\left.25 \mu \mathrm{g} \mathrm{mL}{ }^{-1}\right)$ for various durations (10 minutes, 1 hour and 6 hours). The viability of E.coli after this treatment in dark, visible and UV light conditions is showed in Figure 7 at various incubation times. It was noticed that immediately after exposure (10 minutes), the fraction of viable E.coli declined with in the presence of bare CuONPs and CuONPs/GLYMO/4-HPBA concentrations over $5 \mu \mathrm{g} \mathrm{mL}{ }^{-1}$. After 1 hour of such treatment in dark, visible light and UV light conditions, the viability of E.coli in the presence of nanoparticles was further reduced. After 6 hours incubation with 5-25 $\mu \mathrm{g} \mathrm{mL}^{-1}$ CuONPs/GLYMO/4-HPBA, all E.coli lost completely their viability. Figures 7A, 7B and 7C show that the CuONPs had excellent antibacterial activity towards $E$. coli. There are many various mechanisms discussed in the literature about how CuONPs kill E.coli and their antibacterial action might be a mixture of all of them. One mechanism is based on the photoactive nature of these nanoparticles which in the presence of oxygen from air and visible or UV light, form reactive oxygen species (ROS) which are free radicals and lead to peroxidation of lipids from the bacterial cell membrane. 2,3,5,35

The cell wall of E.coli is negatively charged while the unfunctionalized (bare) CuONPs is positively charged (below $\mathrm{pH}$ 9). Therefore, the un-functionalized CuONPs were able to electrostatically adhere on the bacterial cell surface which led to damage of their cell membrane. When the free CuONPs attach to the cell, the ROS created locally can interact directly with the cell organelles which can amplify the cell damage. The ROS generation begins of a chain of free radical reactions inside the bacteria. Lipid peroxidation is a type of oxidative stress for the bacteria, which leads to its deactivation. 

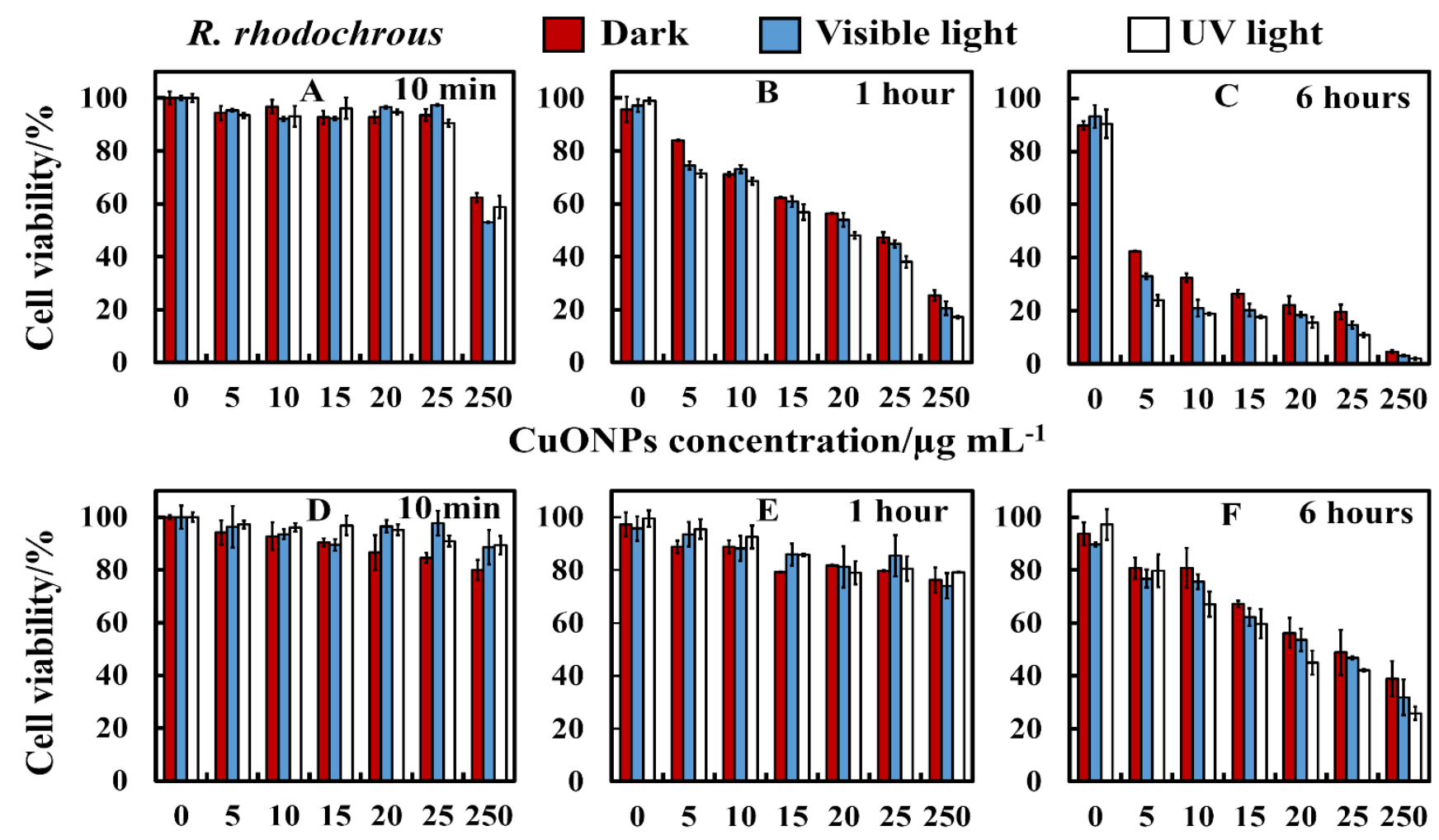

$\mathrm{CuONPs} / \mathrm{GLYMO}$ concentration/ $\mu \mathrm{g} \mathrm{mL} \mathrm{m}^{-1}$
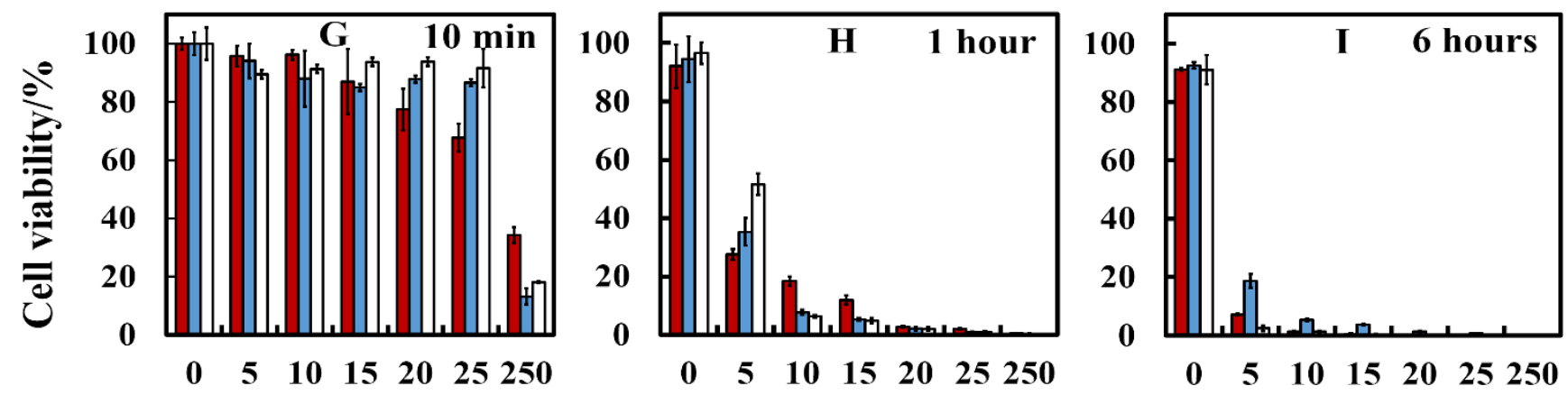

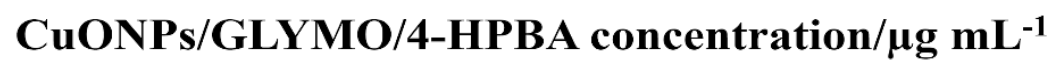

Figure 10. Cell viability of $R$. rhodochrous upon incubation of bare and surface functionalized of CuONPs of different particle concentrations $\left(0,5,10,15,20,25\right.$ and $\left.250 \mu \mathrm{gL}^{-1}\right)$ in dark, visible and UV light conditions. The $R$. rhodochrous cells were incubated with: (A-C) bare CuONPs; (D-F) CuONPs/GLYMO and (G-I) CuONPs/GLYMO/4-HPBA at 10 min, 1 hour and 6 hours exposure times.

However, Figure 7 shows that the antibacterial activity of $25 \mu \mathrm{g}$ $\mathrm{mL}^{-1} \mathrm{CuONPs}$ towards E.coli under UV light for 1 hour is slightly higher than that under dark conditions. This suggests that the ROS generation under UV light has only a minor effect on the antibacterial action of CuONPs.

Another possible antimicrobial mechanism is the release of free $\mathrm{Cu}^{2+}$ ions from the CuONPs which may interfere with the cell membrane proteins. However, the concentration of free $\mathrm{Cu}^{2+}$ ions in the aqueous solution around the CuONPs is negligible due to its very small solubility. The values of the $\mathrm{CuO}$ solubility varies with $\mathrm{pH}$ but in pure water it is approximately $3 \times 10^{-5} \mathrm{M}$.
${ }^{36}$ This is not sufficient to explain the antimicrobial effect of CuONPs, which increases with their concentration, while the $\mathrm{CuO}$ solubility is constant at fixed $\mathrm{pH}$ and temperature).Our working hypothesis is that the strong antimicrobial action can be explained by the direct attraction of the cationic CuONPs with the anionic bacterial cell walls. As CuONPs are aggregates of rough surface, a likely explanation is that their adhesion to the membrane causes its rupture and this is the main contributing factor to the cell death - see below. 

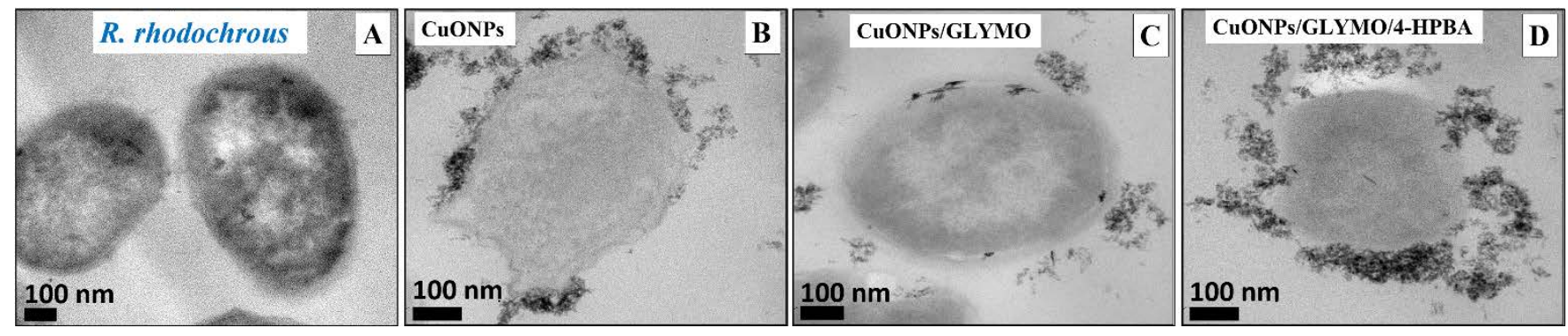

Figure 11. TEM images of $R$. rhodochrous after being incubated for 6 hours into $25 \mu \mathrm{g} \mathrm{mL}^{-1}$ bare and surface functionalized of CuONPs: (A) an untreated sample without CuONPs (B) $R$. rhodochrous incubated with CuONPs (C) R. rhodochrous incubated with CuONPs/GLYMO (D) $R$. rhodochrous incubated with CuONPs/GLYMO/4-HPBA.

We also found that the antibacterial effect of CuONPs/GLYMO (Figure 7D, 7E and 7F) is lower than the one of the bare CuONPs and CuONPs/GLYMO/4-HPBA. Note that CuONP/GLYMO are anionic at this $\mathrm{pH}$ and therefore lack electrostatic adhesion to the bacterial cell walls. Nevertheless, the introduction of a secondary functionalization of these anionic nanoparticles by conjugation of 4-HPBA made the produced CuONPs/GLYMO/4-HPBA much more effective against E.coli than the bare CuONPs. The later effect can be seen in Figure 7G, 7H and 7I. It is interesting that at lower CuONPs/GLYMO/4-HPBA concentrations $\left(5 \mu \mathrm{g} \mathrm{mL}^{-1}\right)$ these anionic particles are several times more effective than the bare CuONPs and CuONPs/GLYMO irrespectively of the time of exposure in dark, visible or UV light conditions. These results require some discussion with respect to the possible factors that may contribute to the antibacterial activity of the CuONPs/GLYMO/4-HPBA. It has been shown that ligands with BA-functionality can covalently bind with diol compounds, like nucleotides, glycate-protein and saccharide. ${ }^{22,}$ ${ }^{23,37}$ Note that despite their negative surface charge, the anionic nanoparticles CuONPs/GLYMO/4-HPBA are showing a very significant antibacterial effect on E.coli even at lower particle concentrations than the bare CuONPs due to their covalent binding to the bacterial membrane. E. coli is surrounded by an outer membrane containing lipopolysaccharides (LPSs) with many diol-groups. ${ }^{37-40}$ The strong (covalent) interactions between the boronic acid terminal group of the CuONPs/GLYMO/4-HPBA particles and the diol-groups from the LPS layer leads to the particle build-up on their cell membranes. In contrast, the adhesion of the bare CuONPs to the bacterial cell membrane is largely driven by electrostatic interactions while the CuONPs/GLYMO/4-HPBA bind to the surface saccharides through formation of boronic ester (see Figure 1). There are many examples in the literature where this effect has been utilized for sensing sugars ${ }^{35,37,41-42}$ but to our best knowledge this is the first time this idea is applied for antibacterial nanoparticle attachment to their targets. Direct CFU/mL measurements (see Figure S8 and S9 and Tables S1 and S2, ESI) also confirm the same trends for the effect of the CuONPs/GLYMO/4-HPBA compared to CuONPs for both E.coli and $R$. rhodochrous. Figures S10 and S11 show the reduction of the CuONPs/GLYMO/4-HPBA required to achieve the same antibacterial effect as CuONPs for these bacteria.

The E.coli samples were sectioned and imaged with SEM and TEM as described in the methods section. Figure 8 and Figure
9 show TEM and SEM images of $E$. coli cells after incubation with CuONPs functionalized with GLYMO and 4-HPBA for up to 6 hours. The images clearly show the adherent layer of nanoparticles which bind to the bacteria. The result was also confirmed via EDX chart of E.coli with CuONPs which revealed the presence of $\mathrm{Cu}$ on the external part of the E.coli surface (Figure S6, ESI).
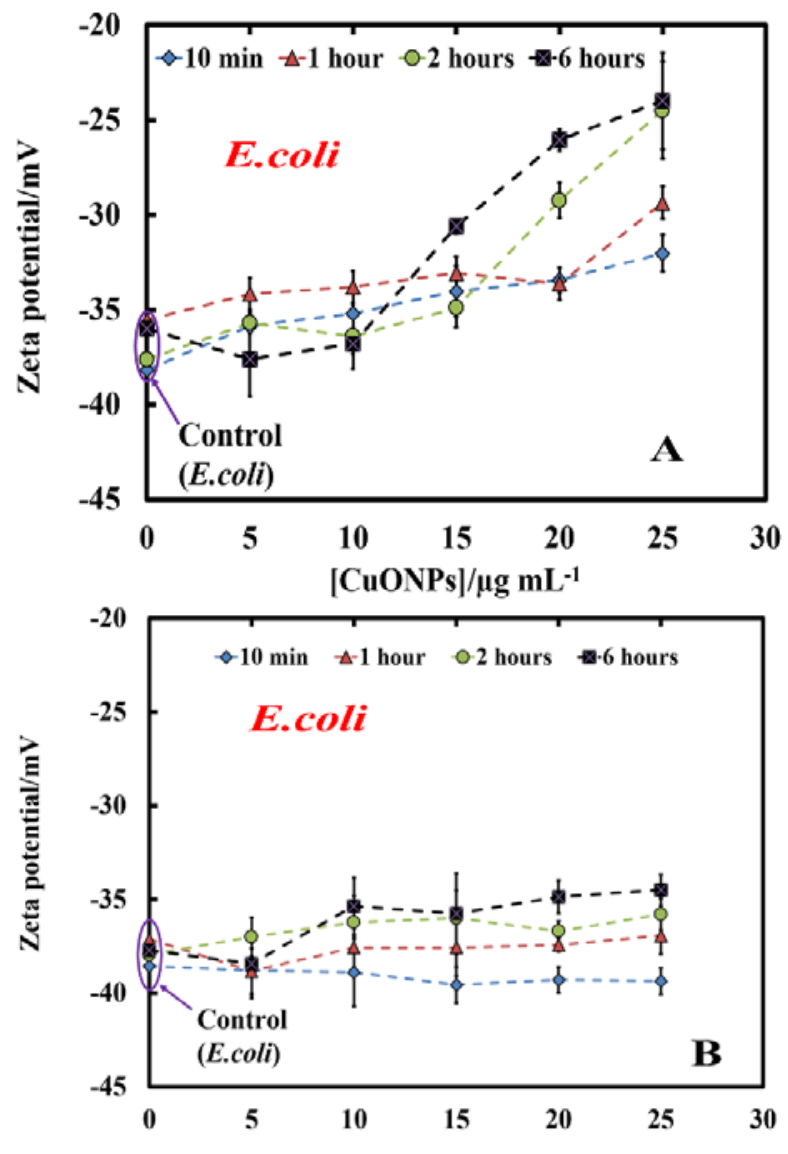

CuONPs/GLYMO/4-HPBA concenteration/ $/ \mathrm{g} \mathrm{mL}^{-1}$

Figure 12. The zeta potential of $E$. coli in aqueous suspensions treated with various concentration of $(\mathrm{A})$ bare $\mathrm{CuONPs}$ and $(\mathrm{B})$ CuONPs/GLYMO/4-HPBA at various exposure times. Error bars indicate standard deviations of means. 

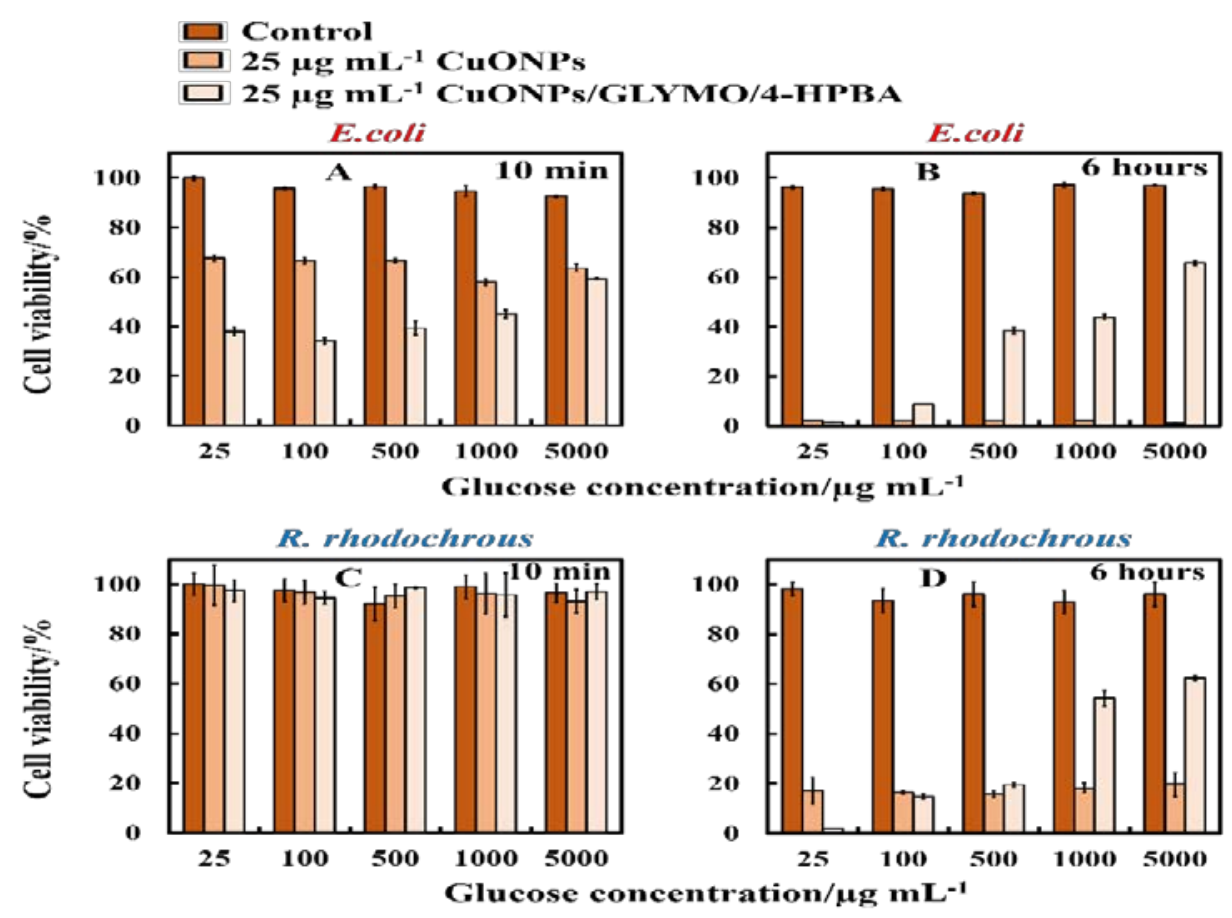

Figure 13. Bacterial cell viability after incubation as a function of nanoparticle concentration for 6 hours at various glucose concentrations (A and B) E.coli and (C and D) R.rhodochrous.

The occasional build-up of more than one layer of CuONPs/GLYMO/4-HPBA particles on the bacteria can also be a result of partial particle aggregation before they bind to the bacterial cell wall. The zeta-potential of the CuONPs is low by magnitude and such partial particle coagulation may take place at various stages of the sample preparation. However, the CuONPs/GLYMO/4-HPBA particles adhere to the negatively charged bacterial cell walls by covalent interactions despite their negative zeta potentials as they dominate the weaker electrostatic repulsion. We confirmed the result by performing EDX on sectioned $E$. coli and compared between bare CuONPs and 4-HPBA functionalized CuONPs which showed presence of $\mathrm{Cu}$ on the outer part of the cell membrane as CuONPs/GLYMO/4-HPBA much higher than the bare CuONPs ones as shown in Figure S13. Our results show higher $\mathrm{Cu}$ concentration on the bacteria outer cell wall for the functionalized CuONPs compared with the bare ones.

\section{Antibacterial Properties of HPBA-Surface} Functionalized CuONPs on $R$. rhodochrous. We also tested the antibacterial properties of the HPBA-modified CuONPs against Gram-positive bacteria. Figure 10 presents the antibacterial assay of $R$. rhodochrous where the control samples of untreated bacteria were compared with the ones treated with bare CuONPs, CuONPs/GLYMO and CuONPs/GLYMO/4HPBA. Note that the cationic bare CuONPs are showing an antibacterial effect on $R$. rhodochrous even at moderate CuONPs concentrations (Figure 10B). We discovered that even at very low concentrations of CuONPs/GLYMO/4-HPBA they are several times more effective against $R$. rhodochrous (Figures $10 \mathrm{H}$ and 10I) than the bare CuONPs (Figure 10B and
10C) and CuONPs/GLYMO (Figure 10E and 10F). A strong effect of the bare CuONPs on $R$. rhodochrous viability was observed only after 6 hours of exposure time (Figure 10C). The charge of the bare CuONPs is an important factor to interact with $R$. rhodochrous membranes, which contribute to their high antibacterial activity.

Note that for exposure times up to 10 minutes and 1 hour (Figure $10 \mathrm{D}$ and $\mathrm{E}$ ), no measurable change in the $R$. rhodochrous viability was detected for CuONPs/GLYMO even at high particle concentrations. This also confirms that potential release of $\mathrm{Cu}^{2+}$ ions is not the main factor in the antibacterial activity of these particles, as CuONPs/GLYMO would support the similar concentration of $\mathrm{Cu}^{2+}$ as the bare CuONPs. We also did not see a significant difference between the samples kept in dark, visible or in UV light conditions at the same CuONPs/GLYMO concentration. TEM imaging shows that the surfaces of $R$. rhodochrous cells accumulate a significant number of deposited nanoparticles after treatment for 6 hours with bare CuONPs (Figure 11B) and CuONPs/GLYMO/4HPBA nanocomposites (Figure 11D). In contrast, the untreated (Figure 11A) and CuONPs/GLYMO treated $R$. rhodochrous (Figure 11C) show smooth and intact $R$. rhodochrous cell membranes.

An additional confirmation for the mechanism of attachment of the CuONPs/GLYMO/4-HPBA to bacterial cells is presented in Figure 12, where we compared the zeta-potential of E. coli after being treated with bare CuONPs and CuONPs/GLYMO/4HPBA of different particle concentrations. Note that when the bacterial cells are treated with bare CuONPs, which are cationic at neutral $\mathrm{pH}$, the zeta potential of the bacteria is reduced by 
absolute value (Figure 12A) due to the partial deposition of the cationic CuONPs on the negatively charged bacterial cell wall.

$\square$ CuONPs

CuONPs/GLYMO/4-HPBA
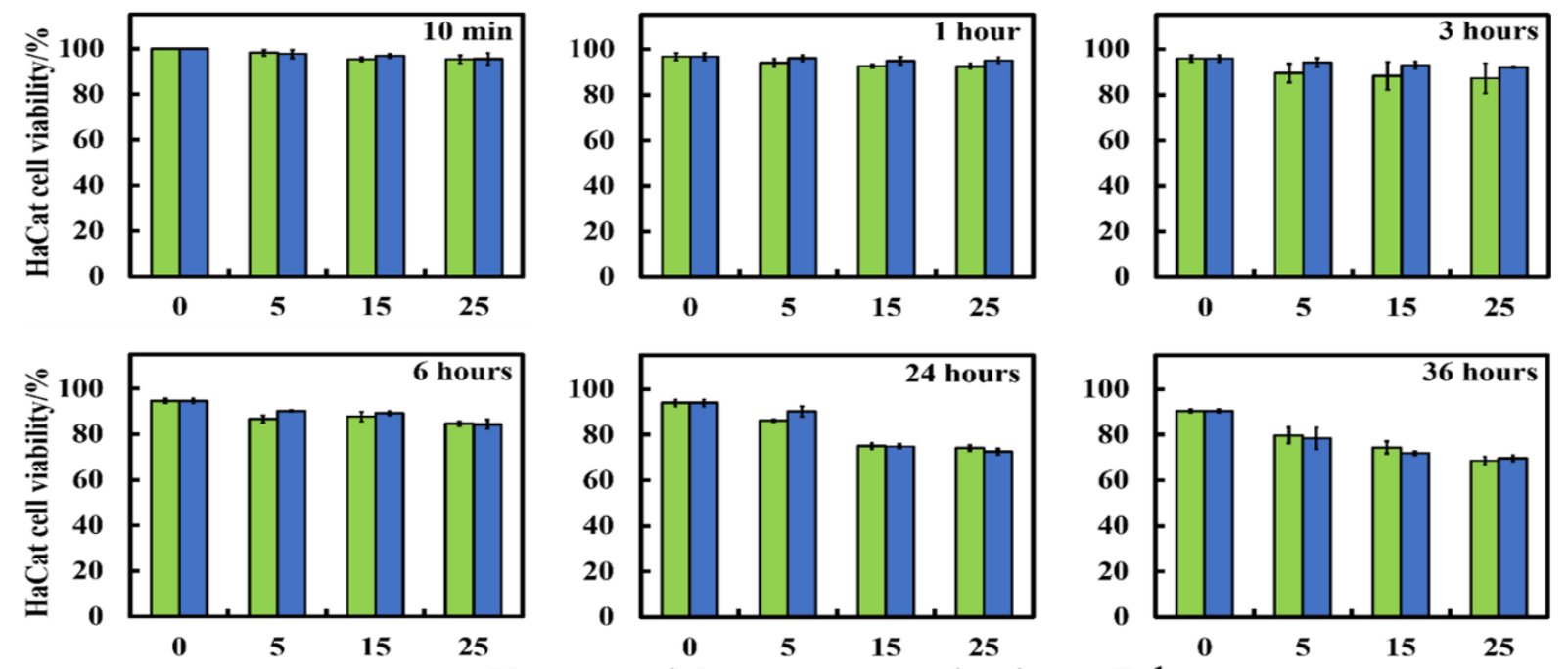

Figure 14. HaCaT cell viability after incubation as a function of nanoparticle concentration for up to 36 hours at with bare $\mathrm{CuONPs}$ and CuONPs/GLYMO/4-HPBA.

However, the incubation of the bacterial cells with CuONPs/GLYMO/4-HPBA does not incur measurable change in their zeta-potential despite their adsorption on the bacterial cell wall (Figure 12B). This is an additional confirmation that the attachment of the CuONPs/GLYMO/4-HPBA to the bacteria is not electrostatic and as Figures 10 and 11 indicate, the BA-functionalized CuONPs bind to the bacteria despite their negative surface charge. This result is easy to understand as the CuONPs/GLYMO/4-HPBA particles are anionic. SEM and TEM images (Figures 8 and 9) confirm the particle deposition of the E.coli outer membrane. These results suggest that the 4-HPBA functional group in the modified CuONPs/GLYMO/4-HPBA particles has a significant role in promoting adhesion to the $R$. rhodochrous membranes. The strong covalent attachment of the CuONPs/GLYMO/4-HPBA with the $R$. rhodochrous cell membrane is likely to be the main contributor towards the bacterial cell membrane disruption and damage which makes it a very efficient antibacterial agent.

Effect of the Presence of Glucose on the Antibacterial Activity of HPBA-Functionalized CuONPs towards E.coli and $\boldsymbol{R}$. rhodochrous Figure 13 shows the antibacterial activity of the HPBA-surface functionalized CuONPs towards E.coli and $R$. rhodochrous at different concentrations of glucose and fixed nanoparticle concentration of $25 \mu \mathrm{g} \mathrm{mL}{ }^{-1}$. Note that all bacteria apparently lost their viability in the presence of bare CuONPs after 6 hours independently of the concentration of glucose in the solution. However, the bacteria viability in the presence of CuONPs/GLYMO/4-HPBA increased with increasing of the glucose concentration. A possible mechanism for this could be that in the presence of glucose the boronic acid functional groups of the CuONPs/GLYMO/4-HPBA nanoparticles bind to the free glucose in solution thus reducing the interaction between 4-HPBA terminal group and the bacterial membranes. This also confirms that the mechanism of attachment of the CuONPs/GLYMO/4-HPBA to the bacteria is based on binding to sugar groups.

Cytotoxicity of Bare and HPBA-Functionalized CuONPs on Human Keratinocytes. Figure 14 shows the cytotoxicity assay of CuONPs and CuONPs/GLYMO/HPBA on HaCaT cells for up to several hours of exposure. The results confirm that $\mathrm{CuONPs/GLYMO/HPBA}$ have negligible toxic effect on these cells while bare CuONPs have some low level of toxicity compared with the control sample. These results are obtained with particle concentrations where they are showing very strong antibacterial effect on E.coli and $R$. rhodochrous while leaving the keratinocyte cells unaffected. We took SEM images of dehydrated HaCaT cells after being treated with bare and HPBA-functionalized CuONPs and compared them with SEM images of the control ssample (no treatment). The results are presented in Figure S12. In both cases, we did not observe significantly different build-up of CuONPs on these images which does not allow directly to differentiate the mechanism of their potential cytotoxic action on keratinocytes. One possible explanation why the skin cells are unaffected by both the bare and the functionalized CuONPs could be that their membrane is easier to bend around the adhering rough nanoparticles and is less prone to dislocation and rupture than the rigid membranes of bacteria. ${ }^{6}$ This result is reassuring that such antimicrobial particles can potentially find application in wound care formulations as an alternative to antimicrobial delivery vehicles. $^{43,44}$

\section{CONCLUSIONS}

In summary, we have developed a novel type of modified CuONPs which have been functionalized with GLYMO and 4HPBA (CuONPs/GLYMO/4-HPBA) to produce an antibacterial agent of much higher efficiency than bare CuONPs. This novel coating with boronic acid functionality 
allows the antimicrobial particles to form covalent bonds with the diol groups from carbohydrates expressed on the cell wall of both Gram-positive and Gram-negative bacteria. We demonstrate the profound differences in the surface properties of the bare CuONPs and the CuONPs/GLYMO/4-HPBA particles which at neutral $\mathrm{pH}$ have different surface charge. The zeta potential of non-functionalized CuONPs, GLYMOfunctionalized CuONPs and 4-HPBA-functionalized CuONPs was $+37 \mathrm{mV},-3 \mathrm{mV}$ and $-10 \mathrm{mV}$, respectively. It was found that both nanoparticles showed opposite surface charge in the aqueous solution at $\mathrm{pH} 6$ as their zeta potential decreased from $+37 \mathrm{mV}$ to $-10 \mathrm{mV}$. Our antibacterial assays showed that the anionic nanoparticles as CuONPs/GLYMO/4-HPBA have much higher antibacterial action than the cationic ones nonfunctionalized CuONPs for both Gram-positive and Gramnegative bacteria. This is explained by the strong adhesion of the anionic particles CuONPs/GLYMO/4-HPBA to the cell walls due to their covalent interactions between the terminal 4Hydroxyphenylboronic acid group and carbohydrates on the cell surface. SEM and TEM images of $R$. rhodochrous and E.coli exposed to 4-HPBA functionalized CuONPs confirmed the formation of a significant build-up of these nanoparticles on the bacterial cell outer membrane. Control experiments proved that the binding ability of the modified CuONPs/GLYMO/4HPBA to bacteria can be adjusted and reversed by adding glucose in the media which engages the boronic acid groups of the CuONPs surface and lessens their ability to attach to bacteria. This effect allows direct control over their antimicrobial action. Preliminary experiments of incubation of the HPBA-functionalized CuONPs with human keratinocytes showed no measurable cytotoxicity. In general, we envisage that this type of functionality can be successfully applied to a range of inorganic nanoparticles, as ZnONPs, $\mathrm{TiO}_{2} \mathrm{NPs}$, $\mathrm{Ag}_{2} \mathrm{ONPs}, \mathrm{Cu}_{2} \mathrm{ONPs}$ and others which would lead to fabrication of superior antimicrobial agents at significantly lower particle concentration.

\section{AUTHOR INFORMATION}

\section{Corresponding Author}

* Phone +44 1482 465660. Email: V.N.Paunov@hull.ac.uk

\section{ORCID}

Ahmed F. Halbus:

0000-0001-9060-7073

Tommy S. Horozov:

0000-0001-8818-3750

Vesselin N. Paunov:

0000-0001-6878-1681

\section{Author Contributions}

The manuscript was written through contributions of all authors. All authors have given approval to the final version of the manuscript.

\section{Funding Sources}

A.F.H acknowledge funding of this work from the Higher Committee for Education Development of Iraq and the University of Babylon, Iraq.

\section{ACKNOWLEDGMENTS}

A.F.H. thanks the Iraqi Government, the Higher Committee for Education Development of Iraq and the University of Babylon, Iraq for the financial support for his PhD study during the work on this project. The authors appreciated the technical help from Tony Sinclair and Ann Lowry at the University of Hull Microscopy Suite with the SEM and TEM sample preparation and imaging. We also thank Zahraa Al-Mashaykhi for her help with FTIR measurements.

\section{ASSOCIATED CONTENT}

In the enclosed electronic supplementary information (ESI) we present the following additional data: (i) The Schematics of the synthesis method of CuONPs; (ii) FTIR analysis of CuONPs calcined at various Temperatures; (iii) XRD pattern of CuONPs annealed at different temperatures; (iv) Effect of the annealing temperature on the particles size and zeta potential of the CuONPs; (v) EDX Diagram of E.coli cells with bare CuONPs (vi) Stability of the zeta-potential of bare and functionalized CuONPs; (vi) CFU assessment of the viability of E. Coli and R. rhodochrous after treatment with bare and functionalized CuONPs; (vi) Antimicrobial efficiency of the bare and HPBAfunctionalized CuONPs; (vii) SEM images of HaCaT cells treated with bare- and HPBA-functionalized CuONPs; (viii) EDX diagrams of the outer surface of E. coli treated with bareandHPBA-functionalized CuONPs

\section{REFERENCES}

(1) Mi, G.; Shi, D.; Wang, M.; Webster, T.J.; Reducing Bacterial Infections and Biofilm Formation Using Nanoparticles and Nanostructured Antibacterial Surfaces, Adv. Healthcare Mater., 2018, 7, 1800103 .

(2) Halbus, A. F.; Horozov, T. S.; Paunov, V. N. Colloid Particle Formulations for Antimicrobial Applications. Adv. Colloid Interface Sci. 2017, 249, 134-148.

(3) Yuan, P.; Ding, X.; Yang, Y.Y.; Xu, Q.-H.; Metal Nanoparticles for Diagnosis and Therapy of Bacterial Infection, Adv. Healthcare Mater., 2018, 7, 1701392.

(4) Alpaslan, E.; Geilich, B.M.; Yazici, H.; Webster, T.J., pHControlled Cerium Oxide Nanoparticle Inhibition of Both GramPositive and Gram-Negative Bacteria Growth, Sci. Rep., 2017, 7, 45859.1-12.

(5) Seil, J.T.; Webster, T.J.; Antimicrobial Applications of Nanotechnology: Methods and Literature, Int. J. Nanomedicine. 2012, 7, 2767-2781.

(6) Penders, J.; Stolzoff, M.; Hickey, D.J.; Andersson, M.; Webster, T.J., Shape-Dependent Antibacterial Effects of Non-Cytotoxic Gold Nanoparticles, Int. J. Nanomedicine, 2017, 12, 2457-2468.

(7) Grigore, M. E.; Biscu, E. R.; Holban, A. M.; Gestal, M. C.; Grumezescu, A. M. Methods of Synthesis, Properties and Biomedical Applications of CuO Nanoparticles. Pharmaceuticals 2016, 9, 75.

(8) Ahamed, M.; Alhadlaq, H. A.; Khan, M.; Karuppiah, P.; Al-Dhabi, N. A. Synthesis, Characterization, and Antimicrobial Activity of Copper Oxide Nanoparticles. J. Nanomater. 2014, 2014, 1-4.

(9) Lazary, A.; Weinberg, I.; Vatine, J.-J.; Jefidoff, A.; Bardenstein, R.; Borkow, G.; Ohana, N. Reduction of Healthcare-Associated Infections in a Long-Term Care Brain Injury Ward By Replacing Regular Linens With Biocidal Copper Oxide Impregnated Linens. Int. J. Infect. Dis. 2014, 24, 23-29.

(10) sani Usman, M. Synthesis, Characterization, and Antimicrobial Properties of Copper Nanoparticles. Int. J. Nanomedicine 2013, 8 , 4467-4479.

(11) Mahapatra, O.; Bhagat, M.; Gopalakrishnan, C.; Arunachalam, K. D. Ultrafine Dispersed CuO Nanoparticles and their Antibacterial Activity. J Exp Nanosci. 2008, 3, 185-193.

(12) Katwal, R.; Kaur, H.; Sharma, G.; Naushad, M.; Pathania, D. Electrochemical Synthesized Copper Oxide Nanoparticles for Enhanced Photocatalytic and Antimicrobial Activity. Ind. Eng. Chem. 2015, 31, 173-184. 
(13) Azam, A.; Ahmed, A. S.; Oves, M.; Khan, M.; Memic, A. SizeDependent Antimicrobial Properties of $\mathrm{CuO}$ Nanoparticles Agains Gram-Positive and-Negative Bacterial Strains. Int. J. Nanomed 2012, 7, 3527-3535.

(14) De Silva, A. P.; Gunaratne, H. N.; Gunnlaugsson, T.; Huxley, A. J.; McCoy, C. P.; Rademacher, J. T.; Rice, T. E. Signaling Recognition Events with Fluorescent Sensors and Switches. Chem. Rev. 1997, 97, 1515-1566.

(15) Lacina, K.; Skládal, P.; James, T. D. Boronic Acids for Sensing and other Applications-A Mini-Review of Papers Published in 2013. Chem. Cent. J. 2014, 8, 1-17.

(16) Trippier, P. C.; McGuigan, C. Boronic Acids in Medicinal Chemistry: Anticancer, Antibacterial and Antiviral Applications. MedChemComm. 2010, 1, 183-198.

(17) Yan, J.; Springsteen, G.; Deeter, S.; Wang, B. The Relationship Among $\mathrm{pKa}, \mathrm{pH}$, and Binding Constants in the Interactions Between Boronic Acids and Diols-it is not as Simple as it Appears. Tetrahedron 2004, 60, 11205-11209.

(18) Kuzimenkova, M. V.; Ivanov, A. E.; Thammakhet, C.; Mikhalovska, L. I.; Galaev, I. Y.; Thavarungkul, P.; Kanatharana, P.; Mattiasson, B. Optical Responses, Permeability and DiolSpecific Reactivity of Thin Polyacrylamide Gels Containing Immobilized Phenylboronic Acid. Polymer 2008, 49, 1444-1454.

(19) Lau, O.-W.; Shao, B.; Lee, M. T. Affinity Mass Sensors: Determination of Fructose. Anal. Chim. Acta 2000, 403, 49-56.

(20) Ertl, P.; Mikkelsen, S. R. Electrochemical Biosensor Array for the Identification of Microorganisms Based on LectinLipopolysaccharide Recognition. Anal. Chem. 2001, 73, 4241-4248.

(21) Wannapob, R.; Kanatharana, P.; Limbut, W.; Numnuam, A.; Asawatreratanakul, P.; Thammakhet, C.; Thavarungkul, P. Affinity Sensor Using 3-aminophenylboronic Acid for Bacteria Detection. Biosens. Bioelectron. 2010, 26, 357-364.

(22) Liu, S.; Wollenberger, U.; Halámek, J.; Leupold, E.; Stöcklein, W.; Warsinke, A.; Scheller, F. W. Affinity Interactions Between Phenylboronic Acid-Carrying Self-Assembled Monolayers and Flavin Adenine Dinucleotide or Horseradish Peroxidase. Chem. Eur.J. 2005, 11, 4239-4246.

(23) Elmas, B.; Onur, M.; SSenel, S.; Tuncel, A. Temperature Controlled RNA Isolation by N-Isopropylacrylamide-Vinylphenyl Boronic Acid Copolymer latex. Colloid. Polym. Sci. 2002, 280, 1137-1146.

(24) DiCesare, N.; Lakowicz, J. R. Evaluation of two Synthetic Glucose Probes for Fluorescence-Lifetime-Based Sensing. Anal. Biochem. 2001, 294, 154-160.

(25) Pandey, V.; Mishra, G.; Verma, S.; Wan, M.; Yadav, R. Synthesis and Ultrasonic Investigations of CuO-PVA Nanofluid. Mater. Sci. Appl. 2012, 3, 664-668.

(26) Grasset, F.; Saito, N.; Li, D.; Park, D.; Sakaguchi, I.; Ohashi, N.; Haneda, H.; Roisnel, T.; Mornet, S.; Duguet, E. Surface Modification of Zinc Oxide Nanoparticles by Aminopropyltriethoxysilane. J. Alloys Compd. 2003, 360, 298-311.

(27) Zhang, D.; Thompson, K.L.; Pelton, R.; Armes, S.P.; Controlling Deposition and Release of Polyol-Stabilized Latex on Boronic AcidDerivatized Cellulose, Langmuir, 2010, 26, 17237-17241.

(28) Pelton, R.; Zhang, D.; Thompson, K.L.; Armes, S.P.; Borate Binding to Polyol-Stabilized Latex, Langmuir, 2011, 27, 21182123.

(29) Cunningham, V. J.; Alswieleh, A. M.; Thompson, K. L.; Williams, M.; Leggett, G. J.; Armes, S. P.; Musa, O. M. Poly(glycerol monomethacrylate)-Poly(benzyl methacrylate) Diblock Copolymer Nanoparticles via RAFT Emulsion Polymerization: Synthesis, Characterization, and Interfacial Activity. Macromolecules 2014, 47, 5613-5623.

(30) Manimaran, R.; Palaniradja, K.; Alagumurthi, N.; Sendhilnathan, S.; Hussain, J. Preparation and Characterization of Copper Oxide Nanofluid for Heat Transfer Applications. Appl. Nanosci. 2014, 4, 163-167.
(31) Luna, I. Z.; Hilary, L. N.; Chowdhury, A. S.; Gafur, M.; Khan, N.; Khan, R. A. Preparation and Characterization of Copper Oxide Nanoparticles Synthesized via Chemical Precipitation Method. OALib. J. 2015, 2, 1-8.

(32) Premazu, P. T.; Stabilization of Rutile $\mathrm{TiO}_{2}$ Nanoparticles with Glymo in Polyacrylic Clear Coating. Mater. Tehnol. 2012, 46, 1924.

(33) Guo, Y.; Chen, Y.; Cao, F.; Wang, L.; Wang, Z.; Leng, Y. Hydrothermal Synthesis of Nitrogen and Boron Doped Carbon Quantum Dots with Yellow-Green Emission for Sensing Cr (VI), Anti-Counterfeiting and Cell Imaging. RSC Adv. 2017, 7, 4838648393.

(34) Arslan, M.; Kiskan, B.; Yagci, Y. Recycling and Self-Healing of Polybenzoxazines with Dynamic Sulfide Linkages. Sci. Rep. 2017, 7, 5207.

(35) Al-Awady, M. J.; Greenway, G. M.; Paunov, V. N. Nanotoxicity of Polyelectrolyte-Functionalized Titania Nanoparticles Towards Microalgae and Yeast: Role of the Particle Concentration, Size and Surface Charge. RSC Adv. 2015, 5, 37044-37059.

(36) Murray, J.V.; Cloke, J.B.; The Solubility of Cupric Oxide in Alkali and the Second Dissociation Constant of Cupric Acid. The Analysis of Very Small Amounts of Copper, J. Am. Chem. Soc., 1936, 58, 2009-2014.

(37) Amin, R.; Elfeky, S. A. Fluorescent Sensor for Bacterial Recognition. Spectrochim. Acta A Mol. Biomol. Spectrosc. 2013, 108, 338-341.

(38) Tümer, M.; Köksal, H.; Sener, M. K.; Serin, S. Antimicrobial Activity Studies of the Binuclear Metal Complexes Derived from Tridentate Schiff Base Ligands. Transition Met. Chem. 1999, 24, 414-420

(39) Imran, M.; Iqbal, J.; Iqbal, S.; Ijaz, N. In Vitro Antibacterial Studies of Ciprofloxacin-Imines and their Complexes with $\mathrm{Cu}$ (II), Ni (II), Co (II), and Zn (II). Turk. J. Biol. 2007, 31, 67-72.

(40) Maillard, A. P. F.; Dalmasso, P. R.; de Mishima, B. A. L.; Hollmann, A. Interaction of Green Silver Nanoparticles with Model Membranes: Possible Role in the Antibacterial Activity. Colloids Surf. B Biointerfaces 2018, 171, 320-326.

(41) Wiskur, S. L.; Lavigne, J. J.; Ait-Haddou, H.; Lynch, V.; Chiu, Y. H.; Canary, J. W.; Anslyn, E. V. p K a Values and Geometries of Secondary and Tertiary Amines Complexed to Boronic Acids Implications for Sensor Design. Org. Lett. 2001, 3, 1311-1314.

(42) Hartley, J. H.; James, T. D.; Ward, C. J. Synthetic Receptors. J. Chem. Soc., Perkin Trans. 1 2000, (19), 3155-3184.

(43) Al-Awady, M. J.; Fauchet, A.; Greenway, G. M.; Paunov, V. N. Enhanced Antimicrobial Effect of Berberine in Nanogel Carriers with Cationic Surface Functionality. J. Mater. Chem. B 2017, 5, 7885-7897.

(44) Al-Awady, M. J.; Weldrick, P. J.; Hardman, M. J.; Greenway, G. M.; Paunov, V. N. Amplified Antimicrobial Action of Chlorhexidine Encapsulated in PDAC-Functionalized Acrylate Copolymer Nanogel Carriers. Mater. Chem. Front. 2018, 2, 20322044.

(45) Lowry, G.V.; Gregory, K.B.; Apte, S.C.; Lead, J.R.; Transformations of Nanomaterials in the Environment. Environ. Sci. Technol. 2012, 46, 6893-6899.

(46) Líbalová, H.; Costa, P. M.; Olsson, M.; Farcal, L.; Ortelli, S.; Blosi, M.; Topinka, J.; Costa, A. L.; Fadeel, B.; Toxicity of SurfaceModified Copper Oxide Nanoparticles in a Mouse Macrophage Cell Line: Interplay of Particles, Surface Coating and Particle Dissolution. Chemosphere. 2018, 196, 482-493.

(47) Chen, K.L.; Bothun, G.D.; Nanoparticles Meet Cell Membranes: Probing Nonspecific Interactions Using Model Membranes. Environ. Sci. Technol. 2014, 48, 873-880.

(48) Perreault, F.; Oukarroum, A.; Melegari, S.P.; Matias, W.G.; Popovic, R.; Polymer Coating of Copper Oxide Nanoparticles Increase Nanoparticles Uptake and Toxicity in the Green Alga Chlamydomonas Reinhardtii. Chemosphere. 2012, 87, 1388-1394. 


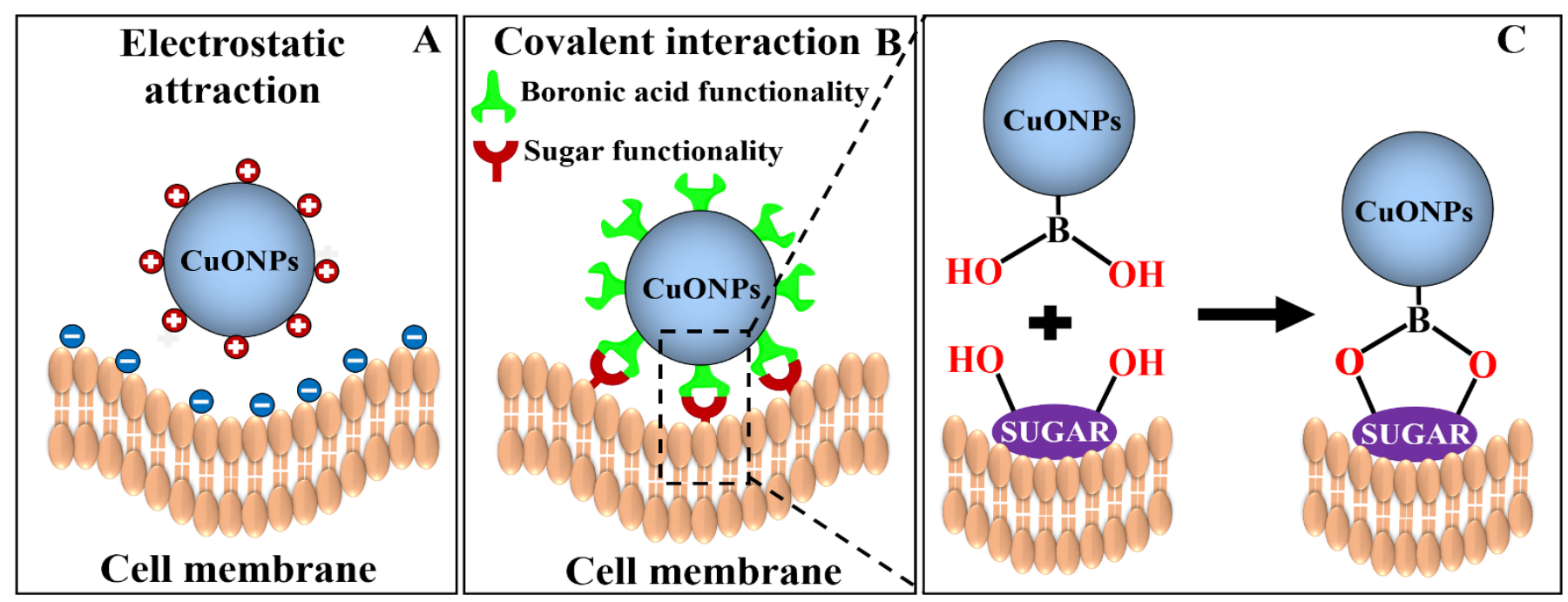

